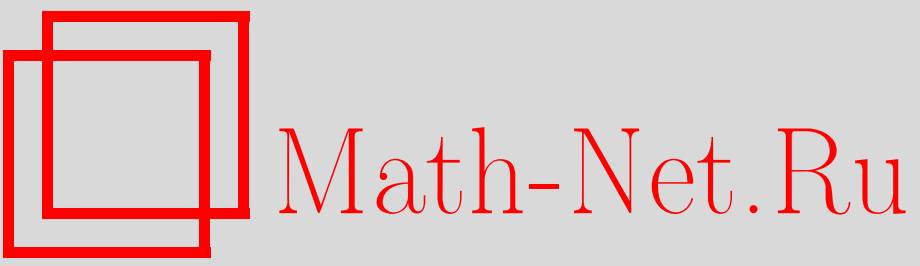

Г. А. Алексеев, О параметризации данными монодромии пространств локальных решений интегрируемых редукций уравнений Эйнштейна, ТMФ, 2005, том 143, номер 2, 278-304

DOI: https://doi.org/10.4213/tmf1815

Использование Общероссийского математического портала Math-Net.Ru подразумевает, что вы прочитали и согласны с пользовательским соглашением

http: //www . mathnet.ru/rus/agreement

Параметры загрузки:

IP : 3.85 .73 .92

26 апреля 2023 г., 05:07:56 


\section{О ПАРАМЕТРИЗАЦИИ ДАННЫМИ МОНОДРОМИИ ПРОСТРАНСТВ ЛОКАЛЬНЫХ РЕШЕНИЙ ИНТЕГРИРУЕМЫХ РЕДУКЦИЙ УРАВНЕНИЙ ЭЙНШТЕЙНА}

Для полей, зависящих только от двух из четырех пространственно-временных координат, пространства локальных решений различных интегрируемых редукций уравнений Эйнштейна представлены как подпространства пространств решений уравнений "нулевой кривизны", выделяемые универсальными (т.е. не зависящими от решений) условиями, накладываемыми на канонические (жордановы) формы искомых матричных функций. Показано, что каждое из этих пространств решений может быть параметризовано конечным набором голоморфных функций спектрального параметра, интерпретируемых как полный набор данных, определяющих монодромию на спектральной плоскости фундаментального решения ассоциированной линейной системы. Показаны однозначная разрешимость прямой и обратной задач такого отображения ( "преобразования монодромии"), т.е. возможность определения данных монодромии для любого локального решения уравнений нулевой кривизны требуемой жордановой формы, а также существование и единственность решения для произвольно выбираемых данных монодромии. Выведены линейные сингулярные интегральные уравнения, решающие обратную задачу. Найден явный вид данных монодромии, отвечающих решениям уравнений Эйнштейна.

Ключевые слова: уравнения Эйнштейна, струнная гравитация, интегрируемость, сингулярные интегральные уравнения, монодромия.

\section{1. ВВЕ ДЕНИЕ}

Для полевых конфигураций, допускающих двумерную абелеву группу пространственно-временных симметрий, уравнения Эйнштейна, как известно, оказываются интегрируемыми в ряде физически интересных случаев. Многие принципиальные вопросы теории гравитации, касающиеся нелинейного характера гравитационных полей и их взаимодействий с материальными полями, можно анализировать, ограничиваясь рассмотрением стационарных полей с осевой симметрией, плоских, цилиндрических и не-

* Математический институт им. В. А. Стеклова РАН, Москва, Россия. E-mail: G.A.Alekseev@mi.ras.ru 
которых других типов волн, а также неоднородных космологических моделей, обладаюших пространственными симметриями, для которых интегрируемость редуцированных полевых уравнений открывает новые возможности для аналитического исследования.

Развитие эффективных методов интегрирования для уравнений Эйнштейна началось с обнаружения того, что эти уравнения для гравитационных полей в вакууме являются интегрируемыми, если метрика пространства-времени зависит только от двух координат и имеет блочно-диагональный вид [1]-[4]. Вслед за этим было показано, что при наличии такой симметрии оказываются интегрируемыми и уравнения Эйнштейна-Максвелла для гравитационных и электромагнитных полей вне их источников [5]-[9], уравнения Эйнштейна-Максвелла-Вейля для гравитационно взаимодействуюших электромагнитного и безмассового двухкомпонентного спинорного полей [10], уравнения Эйнштейна с тензором энергии-импульса безмассового скалярного поля с минимальной связью или идеальной жидкости с предельно жестким уравнением состояния ( $\varepsilon=$ p) [11]. Кроме того, интегрируемыми оказываются и аналогичные редукции уравнений Эйнштейна, описывающих динамику некоторых обобщенных моделей гравитации, например, гравитацию с дилатонным полем в пятимерной теории Калуцы и Клейна [12] или модели струнной гравитации, возникающие в классическом (низкоэнергетическом) пределе бозонного сектора эффективного действия и описываюшие специфическое взаимодействие целого набора безмассовых полей - дилатонного, аксионного, $U(1)$-калибровочных, скалярных (moduli) и гравитационного полей [13]-[17].

Интегрируемость перечисленных выше редуцированных полевых уравнений позволяет развить ряд эффективных методов построения различных семейств их частных решений с любым числом свободных параметров и разнообразной физической интерпреташией, а также сформулировать ряд более обших подходов к изучению широких классов решений ${ }^{1)}$. В частности, в работах [1] было показано, что построение вакуумных решений обшего ("несолитонного") типа сводится к решению матричной задачи Римана-Гильберта, решение которой, в свою очередь, сводится к решению системы линейных сингулярных интегральных уравнений на спектральной плоскости. Для более узкого класса вакуумных и электровакуумных полей - стационарных осесимметричных полей с регулярной осью симметрии - для эффективного построения преобразований из группы внутренних симметрий уравнений Эйнштейна-Максвелла была сформулирована матричная однородная задача Гильберта и построена решающая эту задачу матричная система линейных сингулярных интегральных уравнений [4], [8]. Эта система была впоследствии редуцирована к значительно более простым (не матричным) линейным сингулярным интегральным уравнениям $[21]^{2)}$. В работе [22] для вакуумных полей был предложен некоторый аналог данных рассеяния и соответствующий им дискретный

\footnotetext{
1) Описание многих из этих результатов можно найти в работах [18]-[21].

2) Упрощающее ограничение, использованное в работах [4], [8], [21], накладываемое на пространство рассматриваемых локальных решений и интерпретируемое для осесимметричных полей как условие регулярности оси симметрии, уменьшает вдвое число произвольных функциональных параметров в ядрах соответствующих интегральных уравнений. Такое ограничение оправдано геометрически для осесимметричных полей, однако при рассмотрении полей иной природы, например,
} 
аналог уравнений Гельфанда-Левитана-Марченко.

На основе опыта перечисленных выше подходов и исследования обших свойств многих известных частных решений автором был найден некоторый единый подход к анализу всех описанных выше двумерных редукций уравнений Эйнштейна [23], [20], [24]. Этот подход в каждом случае позволяет рассматривать все пространство локальных решений этих уравнений, не требуя каких-либо дополнительных упрощающих ограничений. Его применение позволяет вычислять новые иерархии семейств частных решений [25] и нелинейные суперпозишии некоторых физически интересных типов полей [26], а также рассматривать различные начальные и граничные задачи [27]. Этот подход, названный автором “методом преобразования монодромии", предполагает, что сначала, в полной аналогии с методом обратной задачи рассеяния, динамическая часть рассматриваемых полевых уравнений переформулируется в виде условий совместности некоторой переопределенной линейной системы со свободным комплексным ( "спектральным") параметром и дополнительными условиями в виде требования сушествования у этой линейной системы матричных интегралов, имеющих определенную структуру ${ }^{3)}$. Затем удивительным образом оказывается, что во всех интегрируемых случаях сушествует такая ассоциированная линейная система, что все монодромные свойства на плоскости спектрального параметра ее фундаментального решения (нормированного на единичную матрицу в некоторой начальной точке пространства-времени) полностью определяются набором не зависящих от координат и голоморфных (в некоторой локальной области спектральной плоскости) функций спектрального параметра.

Возникающий таким образом набор независимых функциональных параметров, называемых данными монодромии, может играть роль, аналогичную данным рассеяния в методе обратной задачи теории рассеяния. Последнее означает, что аналитическая структура этих функций (данных монодромии) однозначно характеризует каждое локальное решение полевых уравнений, так что между всем пространством локальных решений и пространством данных монодромии может быть установлено взаимно однозначное соответствие и могут быть сформулированы прямая и обратная задачи такого отображения ("преобразования монодромии"). Задача определения данных монодромии для заданного локального решения полевых уравнений ("прямая" задача) сводится к отысканию соответствуюшего нормированного фундаментального решения ассоциированной линейной системы с однозначно определенными (после выбора точки нормировки) начальными данными. Для решения "обратной" задачи, т.е. для определения локального решения по произвольно выбранным данным монодромии, была найдена система линейных сингулярных интегральных уравнений. Их правые части и скалярные ядра выражаются соответственно линейно и билинейно через данные монодромии и комплексно-сопряженные им функции. Структура этих интегральных уравнений ока-

сталкивающихся волн, аналогичное ограничение может исключить из рассмотрения некоторые физически интересные типы полевых конфигураций.

3) Для различных упомянутых выше полевых уравнений могут быть различными размерность ассоциированной линейной системы, характер ее особых точек и структура матричных интегралов. 
зывается такой, что для произвольно выбираемых данных монодромии эти уравнения всегда имеют решение, и притом единственное (определенное также локально). По выбранным данным монодромии и соответствующему решению этих интегральных уравнений все компоненты метрики и потенциалов полей выражаются в квадратурах.

Отличительной особенностью изложенного ниже подхода является то, что сначала решается более обшая задача, состоящая из матричных уравнений "нулевой кривизны” с дополнительными условиями, фиксирующими универсальный (т.е. одинаковый для всех решений) вид жордановых форм искомых матричных функций. Построение обшего решения именно этой задачи сводится к решению упомянутой выше системы линейных сингулярных интегральных уравнений, структура которых явно выражается через набор произвольно выбираемых функциональных параметров ( "расширенных данных монодромии"). Общее же решение интересуюших нас редуцированных уравнений Эйнштейна (обобщенных уравнений Эрнста) возникает в результате наложения на эти расширенные данные монодромии простых алгебраических легко разрешаемых связей и определяется вдвое меньшим набором независимых функциональных параметров, называемых просто данными монодромии. В предыдуших работах автора описанная выше конструкция была представлена весьма схематично и лишь для наиболее простых случаев вакуумных уравнений Эйнштейна и электровакуумных уравнений Эйнштейна-Максвелла. Поэтому основной целью настоящей работы является более подробное ее обоснование, включаюшее и некоторые существенные детали, характерные для других интегрируемых редукций уравнений Эйнштейна.

\section{2. ИНТЕГРИРУЕМЫЕ РЕДУКЦИИ УРАВНЕНИЙ ЭЙНШТЕЙНА}

В четырехмерном пространстве-времени, допускающем двумерную абелеву группу изометрий, локальные координаты можно выбрать так, что все компоненты метрики и потенциалы полей будут зависеть только от двух из четырех координат, скажем $x^{\mu}=$ $\left\{x^{1}, x^{2}\right\}$, но не от $x^{a}=\left\{x^{3}, x^{4}\right\}$. Соответствуюшие этой симметрии редукции полевых уравнений могут описывать физически различные типы полей, зависяших либо от времени и одной из пространственных координат ("гиперболический" случай), либо от двух пространственных координат ( "эллиптический” случай). Рассмотрение обоих случаев удобно проводить в единообразной форме, так что об их различиях в выражениях будет напоминать лишь один знаковый символ $\epsilon= \pm 1$ или его "квадратный корень" $j$, так что $\epsilon=1$ и $j=1$ для гиперболических редукций и $\epsilon=-1$ и $j=i$ для эллиптических.

2.1. Выбор координат. В качестве локальных координат $x^{\mu}$ удобно использовать так называемые обобщенные координаты Вейля $\alpha$ и $\beta$, которые определены самой геометрией пространства-времени. Так, функция $\alpha\left(x^{1}, x^{2}\right)$ определяет элемент плошади на двумерных орбитах группы изометрий, причем в силу самих полевых уравнений эта функция является "гармонической", а функция $\beta\left(x^{1}, x^{2}\right)$ определяется как "гармонически" сопряженная ей:

$$
d^{*} d \alpha=0, \quad d \beta=-\epsilon^{*} d \alpha,
$$


где $d$ означает внешнее дифференцирование, a ${ }^{*}$ - дуальнное сопряжение, так что $d{ }^{*} d$ в конформных координатах в эллиптическом случае сводится к двумерному оператору Лапласа, а в гиперболическом - к оператору Д'Аламбера. В некоторых случаях (например, в задаче о столкновении плоских волн или при рассмотрении других начальных или граничных задач) бывает удобно использовать другие координаты, и в этом случае функция $\alpha\left(x^{1}, x^{2}\right)$ становится дополнительной динамической переменной. В качестве координат далее будут использоваться линейные комбинации

$$
\xi=\beta+j \alpha, \quad \eta=\beta-j \alpha,
$$

представляющие собой две вешественные изотропные координаты ( “конусные” переменные) в гиперболическом случае или две комплексно-сопряженные друг другу координаты - в эллиптическом.

2.2. Редуцированные динамические уравнения. Динамические части редуцированных уравнений Эйнштейна для вакуума или электровакуумных уравнений Эйнштейна-Максвелла, а также уравнений Эйнштейна-Максвелла-Вейля удобно представить соответственно в виде уравнений Эрнста [28] или обобщенных уравнений Эрнста [24] для комплексных скалярных функций - потенциалов Эрнста $\mathcal{E}\left(x^{1}, x^{2}\right), \Phi\left(x^{1}, x^{2}\right)$. Наиболее компактно эти уравнения записываются в терминах дифференциальных форм (для простоты знак ^ внешнего произведения дифференциальных форм здесь опушен):

$$
\begin{aligned}
& d^{*} d \mathcal{E}+\frac{d(\alpha+i \delta)}{\alpha} * d \mathcal{E}-\frac{(d \mathcal{E}+2 \bar{\Phi} d \Phi)}{\operatorname{Re} \mathcal{E}+\Phi \bar{\Phi}} * d \mathcal{E}=0, \\
& d^{*} d \Phi+\frac{d(\alpha+i \delta)}{\alpha} * d \Phi-\frac{(d \mathcal{E}+2 \bar{\Phi} d \Phi)}{\operatorname{Re} \mathcal{E}+\Phi \bar{\Phi}} * d \Phi=0 \\
& d^{*} d \alpha=0, \quad d \beta \equiv-\epsilon^{*} d \alpha, \\
& d^{*} d \gamma=0, \quad d \delta \equiv{ }^{*} d \gamma .
\end{aligned}
$$

Спинорное поле здесь описьвается вешественной “гармонической” функцией $\gamma\left(x^{1}, x^{2}\right)$. Эта функция интерпретируется как потенциал для компонент двумерного вектора тока нейтрино. Линейное уравнение для этой функции легко решается, и его общее решение представляется в виде суммы двух функций - произвольных вешественных (в гиперболическом случае) или комплексно-сопряженных друг другу (в эллиптическом случае), одна из которых зависит только от $\xi$, а другая - только от $\eta$. Для электровакуумных полей $\gamma \equiv 0$. Для вакуумных полей имеем также и $\Phi \equiv 0$, так что из всех уравнений остается лиш одно уравнение Эрнста для единственного комплексного потенциала $\mathcal{E}\left(x^{1}, x^{2}\right)$ и уравнение для $\alpha$. Присутствие в системе скалярного поля с минимальной связью, как и идеальной жидкости с предельно жестким уравнением состояния, вообще не сказывается на редуцированных динамических уравнениях и проявляется лишь в уравнениях связи. Решение же этих уравнений связи вычисляется для каждого решения динамических уравнений в квадратурах. Редуцированные уравнения, описывающие в низкоэнергетическом пределе динамику полей в бозонном секторе некоторых моделей теории 
струн и струнной гравитации и включающие аксионное, дилатоное, абелевы калибровочные векторные поля, а также набор скалярных полей, также могут иметь структуру интегрируемых матричных аналогов вакуумного уравнения Эрнста [13]-[15], [17]:

$$
\begin{aligned}
& d^{*} d \mathcal{E}+\frac{d \alpha}{\alpha} * d \mathcal{E}-d \mathcal{E} \cdot(\operatorname{Re} \mathcal{E})^{-1} \cdot{ }^{*} d \mathcal{E}=0, \\
& d^{*} d \alpha=0
\end{aligned}
$$

где $\mathcal{E}\left(x^{1}, x^{2}\right)$ - эрмитова или комплексная симметричная $(d \times d)$-матрица.

2.3. Представление “нулевой кривизны" для интегрируемых редукций уравнений Эйнштейна. Динамические уравнения (1) или (2) можно представить в виде системы уравнений первого порядка (с последующей дополнительной редукцией) для комплексных $(N \times N)$-матриц $\mathbf{U}$ и $\mathbf{V}$, где $N=2$ для вакуумных полей, $N=3$ для полей Эйнштейна-Максвелла или Эйнштейна-Максвелла-Вейля и $N=2 d$ при $d>1$ для моделей струнной гравитации, которым отвечают уравнения (2). Эти матричные уравнения первого порядка состоят из уравнений, имеющих сходный с известным представлением нулевой кривизны вид, и дополнительных условий, требующих, чтобы жордановы формы искомых матричных функций $\mathbf{U}$ и $\mathbf{V}$ имели универсальный (т.е. одинаковый для всех решений) диагональный вид, компоненты которого постоянны, за исключением случая присутствия спинорного поля Вейля, когда они могут зависеть от потенциала этого поля [9], [10], [24], [17]:

$$
\begin{array}{l|l}
\mathbf{U}_{\eta}+\mathbf{V}_{\xi}+\frac{1}{i(\xi-\eta)}[\mathbf{U}, \mathbf{V}]=0, & \begin{array}{l}
\mathbf{U}=\mathcal{F}_{+} \mathbf{U}_{(o)} \mathcal{F}_{+}^{-1}, \\
\mathbf{V}=\mathcal{F}_{-} \mathbf{V}_{(o)} \mathcal{F}_{-}^{-1},
\end{array} \\
\mathbf{U}_{\eta}-\mathbf{V}_{\xi}=0, &
\end{array}
$$

где $\mathcal{F}_{ \pm}$- некоторые матрицы, преобразующие $\mathbf{U}(\xi, \eta)$ и $\mathbf{V}(\xi, \eta)$ к их каноническим формам, а канонические формы $\mathbf{U}_{(\text {o) }}$ и $\mathbf{V}_{(\text {o) }}$ имеют вид

$$
\begin{aligned}
\mathbf{U}_{(o)}^{(N=2)} & =\operatorname{diag}\{i, 0\}, & \mathbf{V}_{(o)}^{(N=2)} & =\operatorname{diag}\{i, 0\}, \\
\mathbf{U}_{(o)}^{(N=3)} & =\operatorname{diag}\left\{i+\mu_{+}(\xi), 0,0\right\}, & \mathbf{V}_{(o)}^{(N=3)} & =\operatorname{diag}\{i+\mu-(\eta), 0,0\}, \\
\mathbf{U}_{(o)}^{(N=2 d)} & =\operatorname{diag}\{\underbrace{i, \ldots, i}_{d}, \underbrace{0, \ldots, 0}_{d}\}, & \mathbf{V}_{(o)}^{(N=2 d)} & =\operatorname{diag}\{\underbrace{i, \ldots, i}_{d} \underbrace{0, \ldots, 0}_{d}\} .
\end{aligned}
$$

Как уже упоминалось выше, $N=2$ для вакуумных полей; наличие электромагнитных полей требует увеличения размерности матрищ $(N=3)$, а присутствие спинорного поля приводит к появлению в выражениях для их собственных значений компонент вектора тока поля нейтрино: $\mu_{+}(\xi)=2 \partial_{\xi} \gamma$ и $\mu_{-}(\eta)=2 \partial_{\eta} \gamma$. В гиперболическом случае функции $\mu_{+}(\xi)$ и $\mu_{-}(\eta)$ должны быть вешественными, а в эллиптическом случае эти функции, как производные от вешественной гармонической функции, должны удовлетворять соотношению $\mu_{+}(\bar{\zeta})=\overline{\mu_{-}(\zeta)}$. Случай $N=2 d$ отвечает струнным моделям, описьваемым матричными уравнениями (2). Случай $d=1$ совпадает со случаем $N=2$, однако 
последний упоминается здесь и всюду ниже отдельно ввиду его классической интерпретации.

Для того чтобы $\mathbf{U}, \mathbf{V}$ (или компоненты $\mathcal{F}_{ \pm}$в $(3)$ ) можно было связать с полевыми переменными и их первыми производными и получить из (3), (4) уравнения Эрнста (1) или (2), компоненты $\mathbf{U}$ и $\mathbf{V}$ должны удовлетворять некоторым дополнительным связям. Ниже эти связи будут представлены в терминах ограничений на фундаментальные решения ассоциированных линейных систем со спектральным параметром, условиями совместности которых являются уравнения (3).

\section{3. СПЕКТРАЛЬНЫЕ ЗАДАЧИ ДЛЯ УРАВНЕНИЙ (3), (4) И (1), (2)}

Уравнения (3) представляют собой условия совместности следующей переопределенной линейной системы порядка $N \times N(N=2,3$ или $2 d)$ :

$$
\begin{aligned}
2 i(w-\xi) \partial_{\xi} \boldsymbol{\Psi} & =\mathbf{U}(\xi, \eta) \boldsymbol{\Psi}, \\
2 i(w-\eta) \partial_{\eta} \boldsymbol{\Psi} & =\mathbf{V}(\xi, \eta) \boldsymbol{\Psi},
\end{aligned}
$$

где $w$ есть комплексный (спектральный) параметр. Для вакуумных и электровакуумных полей эта система была выведена различными методами и использовалась для различных построений в работах [7]-[9]. Описываемый ниже набор дополнительных условий, которые оказываются необходимыми и достаточными для эквивалентности получаемой спектральной задачи уравнениям Эрнста, был найден в работе [23]

3.1. Спектральная задача для уравнений “нулевой кривизны” (3) с условиями (4). Уравнения (3), (4) эквивалентны спектральной задаче, состоящей в одновременном отыскании трех комплексных $(N \times N)$-матричных функций

$$
\boldsymbol{\Psi}(\xi, \eta, w), \quad \mathbf{U}(\xi, \eta), \quad \mathbf{V}(\xi, \eta),
$$

удовлетворяющих системе (5) и таких, что канонические жордановы формы $\mathbf{U}_{(\text {o) }}$ и $\mathbf{V}_{(\text {o) }}$ матриц $\mathbf{U}$ и $\mathbf{V}$ имеют вид (4). Решение именно этой спектральной задачи будет интересовать нас далее в первую очередь, поскольку эта задача непосредственно превращается в спектральную задачу для обобшенных уравнений Эрнста после наложения дополнительных условий, требуюших сушествования матричных интегралов специального вида у систем (5).

3.2. Спектральная задача для обобщенных уравнений Эрнста (1) и (2). Для уравнений Эрнста (1) и (2) спектральная задача формулируется весьма сходным образом как задача одновременного отыскания четырех комплексных $(N \times N)$-матричных функций

$$
\boldsymbol{\Psi}(\xi, \eta, w), \quad \mathbf{U}(\xi, \eta), \quad \mathbf{V}(\xi, \eta), \quad \mathbf{W}(\xi, \eta, w)
$$

первые три из которых удовлетворяют системе (5), и матричные функции $\mathbf{U}$ и $\mathbf{V}$ имеют канонические жордановы формы (4). Еще одним условием этой спектральной задачи является то, что системы (5) должны иметь матричные интегралы определенной 
структуры. В случаях, отвечающих скалярным потенциалам Эрнста $(N=2,3)$, или в матричном случае $(N=2 d, d>1)$ с комплексными симметричными потенциалами Эрнста это есть эрмитовы матричные интегралы $\mathbf{K}(w)$, структура которых содержит матричную функцию $\mathbf{W}$, которая должна быть линейна по спектральному параметру с определенным ниже постоянным матричным коэффициентом $\Omega$ :

$$
\boldsymbol{\Psi}^{\dagger} \cdot \mathbf{W} \cdot \mathbf{\Psi}=\mathbf{K}(w), \quad \mathbf{K}^{\dagger}(w)=\mathbf{K}(w), \quad \frac{\partial \mathbf{W}}{\partial w}=4 i \boldsymbol{\Omega}
$$

Здесь $^{\dagger}$ означает эрмитово сопряжение, причем $\boldsymbol{\Psi}^{\dagger}(\xi, \eta, w)=\overline{\boldsymbol{\Psi}^{\mathrm{T}}(\xi, \eta, \bar{w})}$. Матрица $\boldsymbol{\Omega}$ постоянна и должна иметь следующую структуру:

$$
\boldsymbol{\Omega}^{(N=2)}=\left(\begin{array}{cc}
0 & 1 \\
-1 & 0
\end{array}\right), \quad \boldsymbol{\Omega}^{(N=3)}=\left(\begin{array}{ccc}
0 & 1 & 0 \\
-1 & 0 & 0 \\
0 & 0 & 0
\end{array}\right), \quad \boldsymbol{\Omega}^{(N=2 d)}=\left(\begin{array}{cc}
0 & I_{d} \\
-I_{d} & 0
\end{array}\right)
$$

где $I_{d}$ есть единичная матрица размерности $d \times d$. В матричном случае $(N=2 d, d>1)$ потребуем также сушествования еше одного матричного интеграла $\mathbf{L}(w)$, который в случае комплексных симметричных матричных потенциалов Эрнста должен быть антисимметричным ( ${ }^{\mathrm{T}}$ означает транспонирование матриш):

$$
S \boldsymbol{\Psi}^{\mathrm{T}} \cdot \boldsymbol{\Omega} \cdot \boldsymbol{\Psi}=\mathbf{L}(w), \quad \mathbf{L}^{\mathrm{T}}(w)=-\mathbf{L}(w), \quad S^{2} \equiv(w-\xi)(w-\eta) .
$$

В другом матричном случае $(N=2 d, d>1)$ не с симметричными, а с эрмитовыми матричными потенциалами Эрнста матричный интеграл $\mathbf{K}(w)$ должен быть комплексным симметричным, тогда как матричный интеграл $\mathbf{L}(w)$ должен быть эрмитовым. Матрица $\Omega$ в этом случае должна быть симметричной: $\Omega=\left(\begin{array}{cc}0 & I_{d} \\ I_{d} & 0\end{array}\right)$ (подробнее см. в работе [17]). Поскольку все дальнейшее рассмотрение случаев эрмитовых и симметричных матричных потенциалов Эрнста может проводиться весьма сходным образом, то для простоты мы ограничимся далее лишш случаем симметричных потенциалов.

3.3. Эквивалентность спектральной задачи (4), (5), (8)-(10) уравнениям Эрнста. Нетрудно проверить, что матрица $\mathbf{W}$, появляюшаяся в (8), представляется в виде

$$
\mathbf{W}=4 i(w-\beta) \mathbf{\Omega}+\mathbf{G}(\xi, \eta)
$$

где $\beta=(\xi+\eta) / 2$, а матрица $\mathbf{G}$ - эрмитова. При этом для каждого $N=2,3$ или в симметричном матричном случае $N=2 d$ условия $(4),(5),(8)-(10)$ позволяют выразить компоненты $\mathbf{G}$ через меньший набор функций, а компоненты $\mathbf{U}$ и $\mathbf{V}$ - через эти функции и их первые производные. Эти функции можно отождествить с полевыми переменными и получить для них из условий (4), (5), (8)-(10) уравнения Эрнста (1) или (2). 
3.4. Нормировка решений. Построенные выше спектральные задачи (4), (5), а также (4), (5), (8)-(10) допускают очевидный произвол калибровочных преобразований. Эти преобразования состоят из двух (коммутируюших между собой) групп. Во-первых, это линейные преобразования основных матричных переменных вида

$$
\begin{array}{lll}
\mathbf{U} \rightarrow \mathbf{A} \cdot \mathbf{U} \cdot \mathbf{A}^{-1}, & \mathbf{\Psi} \rightarrow \mathbf{A} \cdot \mathbf{\Psi}, & \mathbf{A}^{\dagger} \cdot \boldsymbol{\Omega} \cdot \mathbf{A}=\boldsymbol{\Omega}, \\
\mathbf{V} \rightarrow \mathbf{A} \cdot \mathbf{V} \cdot \mathbf{A}^{-1}, & \mathbf{W} \rightarrow\left(\mathbf{A}^{-1}\right)^{\dagger} \cdot \mathbf{W} \cdot \mathbf{A}^{-1}, & \mathbf{A}^{\top} \cdot \boldsymbol{\Omega} \cdot \mathbf{A}=\boldsymbol{\Omega},
\end{array}
$$

где постоянная матрица А должна лишь удовлетворять указанным справа соотношени$\mathrm{sм}^{4)}$. Во-вторых, можно выполнять линейные преобразования фундаментального решения линейной системы, при которых изменяются и значения матричных интегралов:

$$
\boldsymbol{\Psi}(\xi, \eta, w) \rightarrow \mathbf{\Psi}(\xi, \eta, w) \cdot \mathbf{C}(w), \quad \| \begin{array}{r}
\mathbf{K}(w) \longrightarrow \mathbf{C}^{\dagger}(w) \cdot \mathbf{K}(w) \cdot \mathbf{C}(w) \\
\mathbf{L}(w) \longrightarrow \mathbf{C}^{\mathrm{T}}(w) \cdot \mathbf{L}(w) \cdot \mathbf{C}(w)
\end{array}
$$

Преобразования (11), отвечающие линейным преобразованиям векторов Киллинга, добавлению постоянных слагаемых к электромагнитным потенциалам, дуальным преобразованиям электромагнитных полей и т.д., можно использовать для приведения значений всех полевых переменных в начальной точке $P_{0}\left(\xi_{0}, \eta_{0}\right)$ к "стандарнтным" начальным значениям, например, совпадаюшим с их значениями для пространства Минковского. Тем самым оказывается фиксированным выбор начального значения для матрицы W:

$$
\mathbf{W}_{0}(w) \equiv \mathbf{W}\left(\xi_{0}, \eta_{0}, w\right)=4 i\left(w-\beta_{0}\right)+\mathbf{G}\left(\xi_{0}, \eta_{0}\right)
$$

Преобразования же $(12)$ со специальным выбором $\mathbf{C}(w)$ используем для того, чтобы нормировать значение $\boldsymbol{\Psi}$ в начальной точке на единичную матрищу:

$$
\boldsymbol{\Psi}(\xi, \eta, w) \rightarrow \boldsymbol{\Psi}(\xi, \eta, w) \cdot \boldsymbol{\Psi}^{-1}\left(\xi_{0}, \eta_{0}, w\right) \quad \Longrightarrow \quad \boldsymbol{\Psi}\left(\xi_{0}, \eta_{0}, w\right) \equiv \mathbf{I}
$$

поскольку именно этот выбор ведет к наиболее простым аналитическим свойствам $\Psi$ на спектральной плоскости. При этом фиксируются значения матричных интегралов $\mathbf{K}(w)$ и $\mathbf{L}(w)$, и условия $(8),(10)$ примут вид

$$
\boldsymbol{\Psi}^{\dagger} \cdot \mathbf{W} \cdot \boldsymbol{\Psi}=\mathbf{W}_{0}(w), \quad \lambda_{+} \lambda_{-} \boldsymbol{\Psi}^{\mathrm{T}} \cdot \boldsymbol{\Omega} \cdot \boldsymbol{\Psi}=\boldsymbol{\Omega}, \quad \frac{\partial \mathbf{W}}{\partial w}=4 i \boldsymbol{\Omega}
$$

где $\lambda_{+}^{2}=(w-\xi) /\left(w-\xi_{0}\right), \lambda_{-}^{2}=(w-\eta) /\left(w-\eta_{0}\right)$, причем $\lambda_{ \pm}(w=\infty)=1$.

Рассмотрим теперь без каких-либо дополнительных упрощающих предположений свойства обшего решения первой из сформулированных выше спектральных задач.

\footnotetext{
${ }^{4)}$ В каждом конкретном случае нетрудно найти обший вид таких матриц. В частности, легко видеть, что при $N=2$ матрица А должна быть вещественной унимодулярной, а при $N=3$, когда $\boldsymbol{\Omega}$ является вырожденной, $\mathbf{A}$ может быть комплексной и иметь более сложный вид.
} 


\section{4. ПРОСТРАНСТВА ЛОКАЛЬНЫХ РЕШЕНИЙ}

Рассмотрим двумерное вешественное многообразие $\mathcal{M}^{2}$ с координатами $(\xi, \eta)$ и выберем в нем некоторую "начальную" точку $P_{0}\left(\xi_{0}, \eta_{0}\right)$. Поскольку все дальнейшее рассмотрение относится только к локальным решениям, в качестве этого многообразия будем рассматривать некоторую окрестность точки $P_{0}$ в $\mathbb{R}^{2}$. Хотя по своему определению координаты $\xi$ и $\eta$ вешественны лишь в гиперболическом случае, а в эллиптическом случае они комплексно сопряжены друг другу, нам будет удобно расширить их область определения, рассматривая в обоих случаях $\xi$ и $\eta$ как две независимые комплексные переменные, пробегающие некоторую окрестность $\Omega^{2}$ точки $P_{0}$ в $\mathbb{C}^{2}$. Введем следуюшие определения.

ОПРЕДЕЛЕНИЕ 1. Набор из двух матричных функций $\mathbf{U}(\xi, \eta)$ и $\mathbf{V}(\xi, \eta)$, голоморфных в некоторой окрестности выбранной начальной точки $P_{0}\left(\xi_{0}, \eta_{0}\right)$, удовлетворяюших в этой окрестности уравнениям (3) и имеюших в каждой точке этой окрестности канонические жордановы формы (4), будем называть локальным решением в точке $P_{0}$ уравнений "нулевой кривизны" с жордановым условием (4), а все множество таких решений - пространством их локальных решений в этой точке.

ОПРЕДЕЛЕниЕ 2. Полный набор полевых переменных (компонет метрики и потенциалов материальных полей, а также соответствуюших им потенциалов Эрнста), которые принимают в выбранной начальной точке $P_{0}\left(\xi_{0}, \eta_{0}\right)$ заданные "стандартные" значения, являются голоморфными по $\xi, \eta$ в некоторой окрестности этой точки и удовлетворяют в этой окрестности полной системе полевых уравнений (например, в форме уравнений Эрнста (1) или (2)), будем называть (нормированным) локальным решением полевых уравнений в точке $P_{0}$, а все множество таких решений - пространством их (нормированных) локальных решений в этой точке.

Без потери общности будем полагать, что окрестность начальной точки $\Omega^{2}$, о которой идет речь в этих определениях, имеет вид $\Omega_{\xi_{0}} \times \Omega_{\eta_{0}}$, где области $\Omega_{\xi_{0}}$ и $\Omega_{\eta_{0}}$ представляют собой окрестности, пробегаемые на комплексной плоскости переменными $\xi$ и $\eta$ вблизи их начальных значений $\xi_{0}$ и $\eta_{0}$, соответственно. Эти области выбираются так, чтобы их образы на спектральной плоскости

$$
\Omega_{+}=\left\{w \mid w=\xi \in \Omega_{\xi_{0}}\right\}, \quad \Omega_{-}=\left\{w \mid w=\eta \in \Omega_{\eta_{0}}\right\}
$$

не пересекались, были выпуклыми и в гиперболическом случае оказывались симметричными относительно вешественной оси, а в эллиптическом случае - симметричными друг другу по отношению к этой оси ${ }^{5}$.

\footnotetext{
5) Напомним, что по определению в областях регулярности решения, которые мы здесь только и рассматриваем, функция $\alpha$ положительна, так что в эллиптическом случае точки $\Omega_{+}$могут лежать лишь выше вещественной оси, а точки $\Omega_{-}-$ниже этой оси. Точки же, где $\alpha=0$, отвечающие различным возможным особенностям геометрии решений (таким, как сингулярности кривизны или
} 
Таким образом, любое локальное решение $\mathbf{U}, \mathbf{V}$ уравнений (3) с условиями (4) голоморфно в некоторой окрестности $\Omega^{2}$ начальной точки:

$$
\mathbf{U}, \mathbf{V}: \Omega^{2} \longrightarrow G L(N, \mathbb{C}), \quad \Omega^{2}=\Omega_{\xi_{0}} \times \Omega_{\eta_{0}} \subset \mathbb{C}^{2},
$$

где $N=2,3$ или $N=2 d, d>1$, в соответствии с размерностью линейной системы. Поэтому аналитические свойства соответствуюшего фундаментального решения $\boldsymbol{\Psi}(\xi, \eta, w)$ линейной системы (5), нормированного условием (14), естественно рассматривать в области $\Omega^{3}$ :

$$
\Psi: \Omega^{3} \longrightarrow G L(N, \mathbb{C}), \quad \Omega^{3}=\Omega^{2} \times \overline{\mathbb{C}} \subset \overline{\mathbb{C}}^{3},
$$

где $\xi$ и $\eta$ пробегают окрестности $\Omega_{\xi_{0}}$ и $\Omega_{\eta_{0}}$ точек $\xi_{0}$ и $\eta_{0}$, соответственно, а спектральный параметр $w$ принимает значения во всей расширенной комплексной плоскости $\overline{\mathbb{C}}$.

\section{5. АНАЛИТИЧЕСКАЯ СТРУКТУРА $\mathbf{\Psi}(\xi, \eta, w)$}

5.1. Особые точки $\boldsymbol{\Psi}(\xi, \eta, w)$ и $\boldsymbol{\Psi}^{-1}(\xi, \eta, w)$. В области $\Omega^{3}$ особым точкам линейной системы (5) отвечают куски комплексных гиперплоскостей $\Omega^{3} \cap\{w=\xi\}$ и $\Omega^{3} \cap$ $\{w=\eta\}$. Нормировка (14) этого решения в начальной точке вносит еше два множества особых точек - куски комплексных гиперплоскостей $\Omega^{3} \cap\left\{w=\xi_{0}\right\}$ и $\Omega^{3} \cap\left\{w=\eta_{0}\right\}$. В соответствии с известными теоремами аналитической теории дифференциальных уравнений в неодносвязной области, которую представляет собой область $\Omega^{3}$ вне ее пересечения с указанными четырьмя комплексными гиперплоскостями, функции $\boldsymbol{\Psi}(\xi, \eta, w)$ и $\Psi^{-1}(\xi, \eta, w)$ являются (неоднозначными) аналитическими функциями ${ }^{6}$.

горизонты Коши и Киллинга), исключаются из нашего локального рассмотрения выбором достаточно малой окрестности $\Omega^{2}$ начальной точки, в которой по условиям нормировки $\alpha_{0} \neq 0$. Однако в некоторых решениях точки $\alpha=0$ отвечают "устранимым" чисто координатным особенностям, таким, как точки оси симметрии в цилиндрических координатах для осесимметричных полей. В этих случаях бывает даже удобно выбирать начальную точку на оси симметрии. При этом области $\Omega_{+}$ и $\Omega_{-}$оказываются перекрывающимися. Однако в этом случае известное условие регулярности оси симметрии суживает пространство локальных решений именно так, что не возникает неоднозначности в соответствующих решениях спектральной задачи, и все последующие построения (с учетом дополнительных связей) легко переносятся на этот подкласс решений.

6) Общие аналитические свойства этих функций, включая зависимость от выбора точки нормировки, были подробно описаны в работе Хаусера и Эрнста [29]. Кроме того, для специального класса полей, обладающих стационарностью и осевой симметрией, в ней также были изучены свойства голоморфной ветви $\boldsymbol{\Psi}(\xi, \eta, w)$ в окрестности регулярных точек оси симметрии. Используемая ниже иная структура разреза не требует каких-либо дополнительных ограничений на вид рассматриваемых полей и пригодна для любого локального решения. Свойства соответствующей этому разрезу голоморфной ветви $\boldsymbol{\Psi}(\xi, \eta, w)$ впервые были описаны и использованы в работах [23], [20]. Затем в работе Хаусера и Эрнста [30] эти свойства детально рассматривались для решений конечной гладкости для гиперболического случая (сталкивающихся плоских волн) и использовались для доказательства общих теорем существования и единственности решений характеристической задачи Коши и доказательства гипотезы Героча для гиперболических уравнений Эрнста. В нижеследующих рассмотрениях используется подход, предложенный в работах [23], [20]. 
5.2. Область голоморфности $\Psi(\xi, \eta, w)$ и $\Psi^{-1}(\xi, \eta, w)$. Для выделения однозначной ветви $\boldsymbol{\Psi}(\xi, \eta, w)$ в области $\Omega^{3}$ необходимо провести разрез, который представлял бы собой гиперповерхность в вешественном шестимерном пространстве, отвечаюшем трехмерной комплексной области $\Omega^{3}$. Этот разрез должен преврашать данную область в односвязную и содержать все четыре указанные выше множества $\Omega^{3} \cap\{w=\xi\}$, $\Omega^{3} \cap\{w=\eta\}, \Omega^{3} \cap\left\{w=\xi_{0}\right\}$ и $\Omega^{3} \cap\left\{w=\eta_{0}\right\}$. Построим этот разрез в виде связной гиперповерхности $\widetilde{\mathcal{L}}$, состоящей из трех линейчатых поверхностей: $\widetilde{\mathcal{L}}=\mathcal{L}_{+} \cup \mathcal{L}_{0} \cup \mathcal{L}_{-}$, где каждая из гиперповерхностей $\mathcal{L}_{+}, \mathcal{L}_{0}$ и $\mathcal{L}_{-}$состоит из прямых, соединяюших на каждой плоскости $\{\xi=$ const, $\eta=$ const $\}$ точки $w=\xi_{0}$ и $w=\xi$, точки $w=\xi_{0}$ и $w=\eta_{0}$ и, наконец, точки $w=\eta_{0}$ и $w=\eta$, соответственно. При необходимости непрерывной деформацией эту гиперповерхность можно превратить в гладкую. Получившаяся в результате такого разреза область $\Omega^{3} \backslash \tilde{\mathcal{L}}$ оказывается односвязной. Действительно, любое сечение разреза $\tilde{\mathcal{L}}$ плоскостью $\{\xi=$ const, $\eta=$ const $\}$ на плоскости спектрального параметра $w$ будет изображаться, очевидно, некоторой непрерьвной кривой $\tilde{L}$, состоящей из трех частей: $\tilde{L}=L_{+} \cup L_{0} \cup L_{-}$. Оставшаяся вне этой кривой часть сферы Римана (расширенной спектральной плоскости) является односвязной, а следовательно, односвязной окажется и область $\Omega^{3} \backslash \tilde{\mathcal{L}}$. Значит, в области $\Omega^{3} \backslash \tilde{\mathcal{L}}$ нормированная функция $\boldsymbol{\Psi}(\xi, \eta, w)$ будет иметь голоморфную ветвь.

Покажем теперь, что на средней части $\mathcal{L}_{0}$ построенного разреза эта ветвь является непрерывной и, следовательно, разрез по $\mathcal{L}_{0}$ можно не проводить, так как эти точки также являются точками голоморфности $\boldsymbol{\Psi}(\xi, \eta, w)$. С этой целью воспользуемся для полученной голоморфной ветви нормированного фундаментального решения $\boldsymbol{\Psi}(\xi, \eta, w)$ факторизациями вида ${ }^{7)}$

$$
\begin{array}{ll|l}
\mathbf{\Psi}(\xi, \eta, w)=\chi_{+}(\xi, \eta, w) \cdot \boldsymbol{\Psi}_{+}(\xi, w), & \boldsymbol{\Psi}_{+}(\xi, w)=\mathbf{\Psi}\left(\xi, \eta_{0}, w\right), \\
\mathbf{\Psi}(\xi, \eta, w)=\chi_{-}(\xi, \eta, w) \cdot \boldsymbol{\Psi}_{-}(\eta, w), & \boldsymbol{\Psi}_{-}(\eta, w)=\mathbf{\Psi}\left(\xi_{0}, \eta, w\right),
\end{array}
$$

где $\boldsymbol{\Psi}_{+}$и $\boldsymbol{\chi}_{+}$являются фундаментальными решениями линейных систем

$$
\left\{\begin{array} { l } 
{ 2 i ( w - \xi ) \partial _ { \xi } \boldsymbol { \Psi } _ { + } = \mathbf { U } ( \xi , \eta _ { 0 } ) \cdot \boldsymbol { \Psi } _ { + } , } \\
{ \boldsymbol { \Psi } _ { + } ( \xi _ { 0 } , w ) = \mathbf { I } , }
\end{array} \quad \left\{\begin{array}{l}
2 i(w-\eta) \partial_{\eta} \chi_{+}=\mathbf{V}(\xi, \eta) \cdot \chi_{+}, \\
\chi_{+}\left(\xi, \eta_{0}, w\right)=\mathbf{I}
\end{array}\right.\right.
$$

а $\boldsymbol{\Psi}_{-}$и $\boldsymbol{\chi}$ - представляют собой фундаментальные решения систем вида

$$
\left\{\begin{array} { l } 
{ 2 i ( w - \eta ) \partial _ { \eta } \boldsymbol { \Psi } _ { - } = \mathbf { V } ( \xi _ { 0 } , \eta ) \cdot \boldsymbol { \Psi } _ { - } , } \\
{ \boldsymbol { \Psi } _ { - } ( \eta _ { 0 } , w ) = \mathbf { I } }
\end{array} \quad \left\{\begin{array}{l}
2 i(w-\xi) \partial_{\xi} \chi_{-}=\mathbf{U}(\xi, \eta) \cdot \chi_{-} \\
\chi_{-}\left(\xi_{0}, \eta, w\right)=\mathbf{I}
\end{array}\right.\right.
$$

Ограничившись для определенности первой из этих факторизаций, заметим, что структура систем (18) позволяет заключить: в области $\Omega^{3}$ матричная функция $\boldsymbol{\Psi}_{+}(\xi, w)$ имеет особые точки $\Omega^{3} \cap\{w=\xi\}$ и $\Omega^{3} \cap\left\{w=\xi_{0}\right\}$, а функция $\chi_{+}(\xi, \eta, w)$ - точки $\Omega^{3} \cap\{w=\eta\}$

\footnotetext{
${ }^{7)}$ Сушествование такой факторизации обусловлено возможностью предельных переходов $\eta \rightarrow$ $\eta_{0}$ и $\xi \rightarrow \xi_{0}$ в первом и во втором уравнениях (5), соответственно, которая вытекает из голоморфности $\boldsymbol{\Psi}(\xi, \eta, w)$ по всем ее аргументам в области $\Omega^{3} \backslash \tilde{\mathcal{L}}$.
} 
и $\Omega^{3} \cap\left\{w=\eta_{0}\right\}$. Поэтому функция $\boldsymbol{\Psi}_{+}(\xi, w)$ будет иметь голоморфную ветвь в области $\Omega^{3} \backslash \mathcal{L}_{+}$, а функция $\chi_{+}(\xi, \eta, w)$ - в области $\Omega^{3} \backslash \mathcal{L}_{-}$. Следовательно, каждая из этих функций, а значит, и их произведение (17) будут голоморфными на $\mathcal{L}_{0}$. Таким образом, нормированное фундаментальное решение ассоциированной линейной системы в области $\Omega^{3}$ с разрезом, состоящим из двух не связанных между собой частей, т.е. в области $\Omega^{3} \backslash \mathcal{L}$, где $\mathcal{L}=\mathcal{L}_{+} \cup \mathcal{L}_{-}$, будет иметь голоморфную ветвь, обозначаемую далее просто как $\boldsymbol{\Psi}(\xi, \eta, w)$ и выделяемую условием нормировки $\boldsymbol{\Psi}\left(\xi_{0}, \eta_{0}, w\right)=\mathbf{I}$.

Построенная голоморфная ветвь $\boldsymbol{\Psi}(\xi, \eta, w)$ будет обладать важным свойством $\boldsymbol{\Psi}(\xi, \eta, w=\infty)=\mathbf{I}$. Действительно, как следует из структуры линейной системы (5), в пределе $w=\infty$ оказывается, что $\partial_{\xi} \boldsymbol{\Psi}=0$ и $\partial_{\eta} \boldsymbol{\Psi}=0$. В силу условий нормировки не зависяшее от координат при $w=\infty$ значение $\boldsymbol{\Psi}$ должно совпадать с единичной матрицей.

Матричная функция $\boldsymbol{\Psi}(\xi, \eta, w)$ в области $\Omega^{3} \backslash \mathcal{L}$ является также голоморфно обратимой. Чтобы убедиться в этом, вычислим детерминант матрицы $\boldsymbol{\Psi}(\xi, \eta, w)$, умножив каждое из уравнений (5) на $\boldsymbol{\Psi}^{-1}$ и взяв след обеих частей. Поскольку $\operatorname{Tr}\left(d \boldsymbol{\Psi} \cdot \boldsymbol{\Psi}^{-1}\right) \equiv$ $d(\ln \operatorname{det} \boldsymbol{\Psi})$, оба полученных соотношения легко интегрируются. С учетом нормировки (14) постоянная интегрирования (зависяшая от $w$ ) вычисляется явно, что приводит к выражениям

$$
\operatorname{det}\|\Psi(\xi, \eta, w)\|= \begin{cases}\lambda_{+}^{-1} \lambda_{-}^{-1} e^{-i\left(\sigma_{+}+\sigma_{-}\right)} & \text {при } N=2,3, \\ \lambda_{+}^{-d} \lambda_{-}^{-d} & \text { при } N=2 d,\end{cases}
$$

где функции $\lambda_{ \pm}(\xi, \eta, w)$ и $\sigma_{ \pm}(\xi, \eta, w)$ имеют вид

$$
\begin{array}{lll}
\lambda_{+}(\xi, w)=\sqrt{\frac{w-\xi}{w-\xi_{0}}}, & \lambda_{+}(\xi, w=\infty)=1, & \sigma_{+}(\xi, w)=\int_{L_{+}} \frac{\mu_{+}\left(\zeta_{+}\right) d \zeta_{+}}{2\left(w-\zeta_{+}\right)} \\
\lambda_{-}(\eta, w)=\sqrt{\frac{w-\eta}{w-\eta_{0}}}, & \lambda_{-}(\eta, w=\infty)=1, & \sigma_{-}(\eta, w)=\int_{L_{-}} \frac{\mu_{-}\left(\zeta_{-}\right) d \zeta_{-}}{2\left(w-\zeta_{-}\right)} .
\end{array}
$$

Из этих выражений видно, что детерминант $\boldsymbol{\Psi}(\xi, \eta, w)$ отличен от нуля во всех точках области $\Omega^{3} \backslash\left(\mathcal{L}_{+} \cup \mathcal{L}_{-}\right)$и, следовательно, $\Psi^{-1}(\xi, \eta, w)$ также голоморфна в этой области.
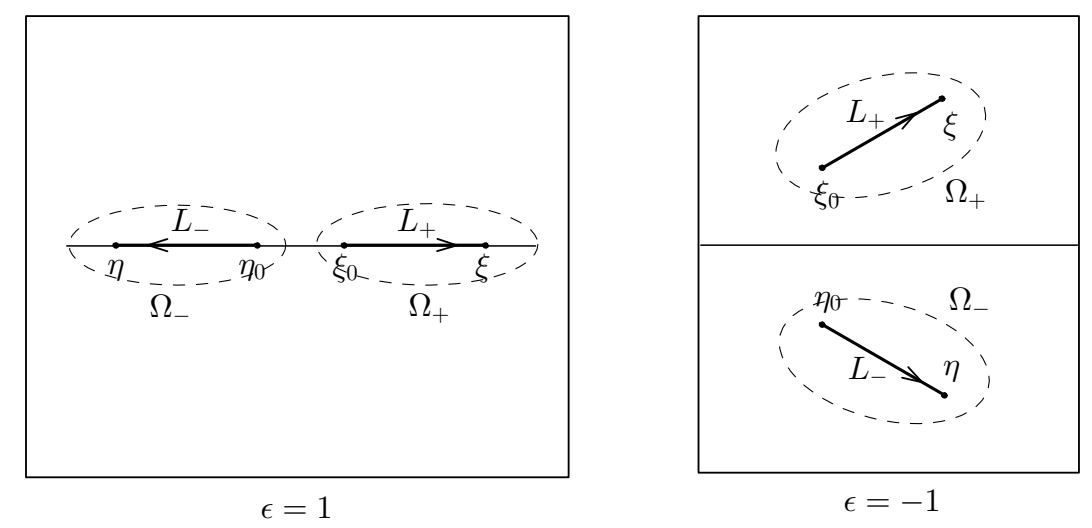
Типичное сечение области $\Omega^{3} \backslash \mathcal{L}$ плоскостью $\{\xi=$ const, $\eta=$ const $\}$, называемое нами просто спектральной плоскостью, изображено на рисунке, где как для гиперболического $(\epsilon=1)$, так и для эллиптического $(\epsilon=-1)$ случаев показана структура локальных областей $\Omega_{ \pm}(16)$, ограниченных штриховыми линиями, и содержашихся в них локальных разрезов $L_{+}$и $L_{-}$, которым для дальнейшего удобства придана определенная ориентация, а именно, кривая $L_{+}$идет из точки $w=\xi_{0}$ в точку $w=\xi$, а $L_{-}-$из точки $w=\eta_{0}$ в точку $w=\eta$. Заметим, что в гиперболическом случае для различных областей пространства-времени относительное расположение на вешественной оси точек $w=\xi_{0}$ и $w=\xi$ и, аналогично, точек $w=\eta_{0}$ и $w=\eta$ может отличаться от указанного на рисунке.

5.3. Ветвления $\boldsymbol{\Psi}(\xi, \eta, w)$ и $\boldsymbol{\Psi}^{-1}(\xi, \eta, w)$ на "концах" разрезов $\mathcal{L}_{+}$и $\mathcal{L}_{-}$. В силу того, что канонические жордановы формы матриц $\mathbf{U}$ и $\mathbf{V}$ имеют вид (4), точки $\xi=w$ и $\eta=w$ являются соответственно для $\boldsymbol{\Psi}_{+}(\xi, w)$ и $\boldsymbol{\Psi}_{-}(\eta, w)$, а следовательно (в силу факторизаций $(17))$, и для $\boldsymbol{\Psi}(\xi, \eta, w)$ точками ветвления. В окрестностях этих точек, т.е. для каждого значения $w \in \Omega_{+}$и значений $\xi$, достаточно близких к $w$, а также для каждого значения $w \in \Omega_{-}$и значений $\eta$, достаточно близких к этим $w$, не нормированные пока фундаментальные решения (5) могут быть представлены соответственно сходящимися в некоторых окрестностях рядами

$$
\begin{aligned}
\mathbf{\Psi}(\xi, \eta, w)= & (\xi-w)^{r_{+}}\left[a_{0}+a_{1}(\xi-w)+a_{2}(\xi-w)^{2}+\cdots\right]+ \\
& +b_{0}+b_{1}(\xi-w)+b_{2}(\xi-w)^{2}+\cdots, \\
\Psi(\xi, \eta, w)= & (\eta-w)^{r_{-}}\left[c_{0}+c_{1}(\eta-w)+c_{2}(\eta-w)^{2}+\cdots\right]+ \\
& +d_{0}+d_{1}(\eta-w)+d_{2}(\eta-w)^{2}+\cdots,
\end{aligned}
$$

где все коэффициенты в первом разложении являются голоморфными функциями параметров $\eta$ и $w$, а во втором - параметров $\xi$ и $w$, поскольку эти коэффициенты алгебраически выражаются через величины $\mathbf{U}(\xi=w, \eta)$ и $\mathbf{V}(\xi, \eta=w)$, соответственно, и их производные по $w$. Показатели $r_{+}$и $r_{-}$в этих разложениях определяются собственными значениями матриц $\mathbf{U}$ и $\mathbf{V}$ (с учетом множителей $2 i$ в левых частях уравнений $(5))$ и оба оказываются равными $-1 / 2$ при отсутствии спинорного поля $\left(\mu_{+}(\xi) \equiv 0, \mu_{-}(\eta) \equiv 0\right)$ или

$$
-\frac{1}{2}+\frac{i}{2} \mu_{+}(w), \quad-\frac{1}{2}+\frac{i}{2} \mu_{-}(w)
$$

соответственно, при его наличии. Нетрудно показать также, что для функции $\boldsymbol{\Psi}^{-1}(\xi, \eta, w)$ имеют место разложения, аналогичные (21), в которых, однако, следует заменить $r_{+} \rightarrow-r_{+}$и $r_{-} \rightarrow-r_{-}$.

Ограничивая области $\Omega_{\xi_{0}}$ и $\Omega_{\eta_{0}}$ изменения переменных $\xi$ и $\eta$ достаточно малыми окрестностями их начальных значений $\xi_{0}$ и $\eta_{0}$, можно добиться того, что точки $\xi=\xi_{0}$ и $\eta=\eta_{0}$ будут лежать внутри кругов сходимости рядов (21) для $\boldsymbol{\Psi}(\xi, \eta, w)$ и аналогичных разложений для $\boldsymbol{\Psi}^{-1}(\xi, \eta, w)$. Поскольку в этом случае весь разрез $\mathcal{L}_{+}$оказывается в круге сходимости первого разложения в $(21)$, а разрез $\mathcal{L}_{-}{ }^{-}$в 
круге сходимости второго из этих разложений, то, используя эти разложения в выражении (14), можно вычислить соответствующие разложения для единственного нормированного фундаментального решения. Если при этом учесть, что это решение, как мы уже знаем из предыдушего, должно быть голоморфно в $\Omega^{3}$ вне разрезов $\mathcal{L}_{+}$и $\mathcal{L}_{-}$, то получим, что в окрестности $\mathcal{L}_{ \pm}$нормированное фундаментальное решение $\boldsymbol{\Psi}(\xi, \eta, w)$ и функция $\boldsymbol{\Psi}^{-1}(\xi, \eta, w)$ представляются в виде

$$
\begin{aligned}
& \mathcal{L}_{+}: \quad \Psi(\xi, \eta, w)=A\left(\frac{\xi-w}{\xi_{0}-w}\right)^{r_{+}}+B, \quad \Psi^{-1}(\xi, \eta, w)=\tilde{A}\left(\frac{\xi-w}{\xi_{0}-w}\right)^{-r_{+}}+\widetilde{B} \\
& \mathcal{L}_{-}: \quad \boldsymbol{\Psi}(\xi, \eta, w)=C\left(\frac{\eta-w}{\eta_{0}-w}\right)^{r_{-}}+D, \quad \Psi^{-1}(\xi, \eta, w)=\widetilde{C}\left(\frac{\eta-w}{\eta_{0}-w}\right)^{-r_{-}}+\widetilde{D}
\end{aligned}
$$

где функции $A(\xi, \eta, w), B(\xi, \eta, w), \tilde{A}(\xi, \eta, w)$ и $\widetilde{B}(\xi, \eta, w)$ голоморфны в окрестности разреза $\mathcal{L}_{+}$, а функции $C(\xi, \eta, w), D(\xi, \eta, w), \widetilde{C}(\xi, \eta, w)$ и $\widetilde{D}(\xi, \eta, w)$ - в окрестности разреза $\mathcal{L}_{-}$. Заметим, что полученные ранее выражения для детерминанта $\boldsymbol{\Psi}$ подсказывают полезную модификацию выражений (22). Так, переопределив голоморфные коэффициенты в выражениях (22), ветвящиеся множители в них можно заменить на новые, выражающиеся через функции $\lambda_{ \pm}$и $\sigma_{ \pm}$, определенные в (20):

$$
\begin{aligned}
& \mathcal{L}_{+}: \quad \boldsymbol{\Psi}=\lambda_{+}^{-1} e^{-i \sigma_{+}} A+B, \quad \mathcal{L}_{-}: \quad \boldsymbol{\Psi}=\lambda_{-}^{-1} e^{-i \sigma_{-}} C+D, \\
& \boldsymbol{\Psi}^{-1}=\lambda_{+} e^{i \sigma_{+}} \tilde{A}+\widetilde{B}, \quad \boldsymbol{\Psi}^{-1}=\lambda_{-} e^{i \sigma_{-}} \widetilde{C}+\widetilde{D} .
\end{aligned}
$$

Впоследствии мы уточним структуру коэффициентов в этих локальных представлениях, однако уже из выражений (23) видно, что на спектральной плоскости, т.е. при заданных $\xi$ и $\eta$, во внутренних точках контуров $L_{+}$и $L_{-}$функции $\boldsymbol{\Psi}$ и $\boldsymbol{\Psi}^{-1}$ имеют конечные пределы слева и справа. Полуразности этих пределов (т.е. скачки каждой из этих функций на контурах) удовлетворяют условию Гёльдера. В случае отсутствия спинорного поля $\left(\mu_{+}(\xi) \equiv 0, \mu_{-}(\eta) \equiv 0\right)$ имеем $r_{+}=r_{-}=-1 / 2$, поэтому в начальных точках $w=\xi_{0}$ и $w=\eta_{0}$ контуров $L_{+}$и $L_{-}$скачки $\boldsymbol{\Psi}(\xi, \eta, w)$ стремятся к нулю, а в их конечных точках $w=\xi$ и $w=\eta$ обрашаются в бесконечность, но интегрируемым образом. Скачки функции $\boldsymbol{\Psi}^{-1}(\xi, \eta, w)$, наоборот, имеют интегрируемые бесконечности в начальных точках $w=\xi_{0}$ и $w=\eta_{0}$ контуров $L_{+}$и $L_{-}$, а в их конечных точках $w=\xi$ и $w=\eta$ стремятся к нулю. При наличии спинорного поля в гиперболическом случае его вклад в величины $r_{+}$и $r_{-}$чисто мнимый, так что в этом случае поведение скачков функций $\boldsymbol{\Psi}$ и $\boldsymbol{\Psi}^{-1}$ на концах разрезов качественно не изменяется. Однако в эллиптическом случае это уже не так, и для интегрируемости скачков этих величин на концах разрезов требуется наложить некоторые ограничения на характеристики спинорного поля, чтобы обеспечить выполнение условий $\left|\operatorname{Re} r_{+}\right| \leqslant 1$ и $\left|\operatorname{Re} r_{-}\right| \leqslant 1$. Описанные выше свойства $\Psi$ и $\boldsymbol{\Psi}^{-1}$ в области $\Omega^{3}$ можно сформулировать в виде теоремы, имеющей очевидное, но важное для дальнейшего следствие. 
TЕОРема 1. Для любого локального решения $\mathbf{U}(\xi, \eta), \mathbf{V}(\xi, \eta)$ уравнений “нулевой кривизнь” (3) с эсордановым условием (4) в $\mathbb{C}^{2}$ найдется окрестность $\Omega^{2}=\Omega_{\xi_{0}} \times$ $\Omega_{\eta_{0}}$ начальной точки $P_{0}$, в которой существует единственное фундаментальное решение $\boldsymbol{\Psi}(\xi, \eta, w)$ линейной системь (5) с условием (4) такое, что:

1) $\boldsymbol{\Psi}(\xi, \eta, w)$ является голоморфницм в области $\left(\Omega^{2} \times \overline{\mathbb{C}}\right) \backslash\left(\mathcal{L}_{+} \cup \mathcal{L}_{-}\right)$;

2) $\boldsymbol{\Psi}\left(\xi_{0}, \eta_{0}, w\right)=\mathbf{I} u \mathbf{\Psi}(\xi, \eta, w=\infty)=\mathbf{I}$

3) $\boldsymbol{\Psi}(\xi, \eta, w)$ имеет на разрезах $L_{+}$и $L_{-}$конечные предель справа и слева, разность которых удовлетворяет условию Гёльдера и интегрируема на концах (в әллиптическом случае последнее верно только при ограничении на спинорные поля вида $\left|\operatorname{Im} \mu_{+}(\xi)\right|<1$ или, эквивалентно, $\left.\left|\operatorname{Im} \mu_{-}(\eta)\right|<1\right)$;

4) всеми этими свойствами обладает и функиия $\Psi^{-1}(\xi, \eta, w)$.

СледСтвиЕ 1. В области $\left(\Omega^{2} \times \overline{\mathbb{C}}\right) \backslash\left(\mathcal{L}_{+} \cup \mathcal{L}_{-}\right)$как нормированное фундаментальное решение $\boldsymbol{\Psi}(\xi, \eta, w)$ линейной системы (5) с условием (4), так и обратная ему матричная функиия $\boldsymbol{\Psi}^{-1}(\xi, \eta, w)$ могут быть представлень интегралами типа Коши от скачков этих функиий на составном разрезе $L=L_{+} \cup L_{-}$спектральной плоскости:

$$
\boldsymbol{\Psi}=\mathbf{I}+\frac{1}{i \pi} \int_{L} \frac{[\Psi]_{\zeta}}{\zeta-w} d \zeta, \quad \Psi^{-1}=\mathbf{I}+\frac{1}{i \pi} \int_{L} \frac{\left[\boldsymbol{\Psi}^{-1}\right]_{\zeta}}{\zeta-w} d \zeta
$$

Здесь $w$ лежит вне $L$, переменная $\zeta$ пробегает разрез $L$, состояший из двух непересекаюшихся разрезов $L_{+}$и $L_{-}$, а $[\ldots]_{\zeta}$ означает скачок функции, т.е. полуразность ее предельных значений слева и справа от $L$ в точке $\zeta \in L$.

\section{6. ЛОКАЛЬНАЯ СТРУКТУРА $\Psi(\xi, \eta, w)$ В ОКРЕСТНОСТИ РАЗРЕЗОВ $\mathcal{L}_{+}$И $\mathcal{L}_{-}$}

Прямым следствием универсальной структуры (4) жордановых форм $\mathbf{U}(\xi, \eta)$ и $\mathbf{V}(\xi, \eta)$ является то, что среди линейно независимых решений, линейными комбинациями которых являются столбцы матришы нормированного фундаментального решения $\Psi(\xi, \eta, w)$, при $N=2,3$ имеется всего одно, а при $N=2 d$ имеется $d$ решений, ветвяшихся в окрестности точек $\xi=w$, тогда как остальные решения не имеют ветвления в этих точках. Такое же число ветвяшихся и неветвящихся решений имеется и в окрестности $\eta=w$. Рассмотрим теперь два вспомогательных определенных локально-фундаментальных решения $\boldsymbol{\Psi}_{(+)}$и $\boldsymbol{\Psi}_{(-)}$. В первом из них, определенном в окрестности $\xi=w$, при $N=2,3$ выберем ветвяшееся решение в качестве первого столбца, тогда как остальные столбшы будут состоять только из неветвяшихся решений. При $N=2 d$ ветвяшиеся решения будут занимать первые $d$ столбцов, а $d$ последних будут образованы неветвяшимися решениями. Другое фундаментальное решение определяется локально в окрестности $\eta=w$ и строится аналогично из решений, ветвяшихся и неветвяшихся в этих точках. При этом аналогичное разделение по характеру ветвления произойдет и в строках матриц, обратных к этим фундаментальным решениям. В окрестности $\xi=w$ структуру этих решений 
при $N=3$ можно изобразить наглядно следующим образом (случай $N=2$ вполне аналогичен, и в нем надо лишь опустить третьи строки и столбцы матриц):

$$
\begin{aligned}
& \Psi_{(+)}=\frac{1}{\Sigma_{+}}\left(\begin{array}{l}
* \\
* \\
*
\end{array}\right) \otimes(1,0,0)+\left(\begin{array}{lll}
0 & * & * \\
0 & * & * \\
0 & * & *
\end{array}\right), \\
& \Psi_{(+)}^{-1}=\Sigma_{+}\left(\begin{array}{l}
1 \\
0 \\
0
\end{array}\right) \otimes\left(\begin{array}{lll}
* & * & *
\end{array}\right)+\left(\begin{array}{lll}
0 & 0 & 0 \\
* & * & * \\
* & * & *
\end{array}\right),
\end{aligned}
$$

где $\Sigma_{(+)}=(\xi-w)^{-r_{+}}$, знак $\otimes$ есть тензорное произведение, а $*$ обозначает компоненты, голоморфные в окрестности $\xi=w$. При $N=2 d$ структура этих матриц изменяется, как показано ниже для $d=2$ :

$$
\begin{aligned}
& \Psi_{(+)}=(\xi-w)^{-1 / 2}\left(\begin{array}{ll}
* & * \\
* & * \\
* & * \\
* & *
\end{array}\right) \otimes\left(\begin{array}{llll}
1 & 0 & 0 & 0 \\
0 & 1 & 0 & 0
\end{array}\right)+\left(\begin{array}{llll}
0 & 0 & * & * \\
0 & 0 & * & * \\
0 & 0 & * & * \\
0 & 0 & * & *
\end{array}\right), \\
& \Psi_{(+)}^{-1}=(\xi-w)^{1 / 2}\left(\begin{array}{ll}
1 & 0 \\
0 & 1 \\
0 & 0 \\
0 & 0
\end{array}\right) \otimes\left(\begin{array}{llll}
* & * & * & * \\
* & * & * & *
\end{array}\right)+\left(\begin{array}{llll}
0 & 0 & 0 & 0 \\
0 & 0 & 0 & 0 \\
* & * & * & * \\
* & * & * & *
\end{array}\right),
\end{aligned}
$$

где $\otimes$ следует понимать как обычное матричное умножение или как тензорное умножение, однако в последнем случае все $(d \times 2 d)$-, $(2 d \times d)$ - и $(2 d \times 2 d)$-матрицы следует рассматривать как двумерные, но имеюшие своими компонентами блоки размерностью $d \times d$. Второе вспомогательное фундаментальное решение $\boldsymbol{\Psi}_{(-)}$имеет аналогичную локальную структуру в окрестности $\eta=w$. Локальные структуры этих решений можно записать в виде

$$
\begin{array}{lll}
\mathcal{L}_{+}: & \boldsymbol{\Psi}_{(+)}=\frac{1}{\Sigma_{+}} \psi_{(+)} \otimes \mathbf{k}_{(+)}+\mathbf{M}_{(+)}, & \boldsymbol{\Psi}_{(+)}^{-1}=\Sigma_{+} \mathbf{l}_{(+)} \otimes \boldsymbol{\varphi}_{(+)}+\mathbf{N}_{(+)} \\
\mathcal{L}_{-}: & \boldsymbol{\Psi}_{(-)}=\frac{1}{\Sigma_{-}} \Psi_{(-)} \otimes \mathbf{k}_{(-)}+\mathbf{M}_{(-)}, & \boldsymbol{\Psi}_{(-)}^{-1}=\Sigma_{-} \mathbf{l}_{(-)} \otimes \boldsymbol{\varphi}_{(-)}+\mathbf{N}_{(-)}
\end{array}
$$

где компоненты столбцов (или $(2 d \times d)$-матриц) $\boldsymbol{\psi}_{( \pm)}$, строк (или $(d \times 2 d)$-матриц) $\boldsymbol{\varphi}_{( \pm)}$ и матриц $\mathbf{M}_{ \pm}$и $\mathbf{N}_{ \pm}$голоморфны на соответствуюшем разрезе, а их структура (расположение нулей и единиц) легко прочитывается из представления (25) или аналогичного представления $\boldsymbol{\Psi}_{(-)}$и $\boldsymbol{\Psi}_{(-)}^{-1}$. Это позволяет нам установить, что входящие в выражения (26) фрагменты алгебраической структуры этих вспомогательных фундаментальных решений должны удовлетворять на соответствуюших разрезах алгебраическим соотношениям

$$
\begin{aligned}
& \mathcal{L}_{+}: \quad \mathbf{M}_{(+)} \cdot \mathbf{l}_{(+)}=0, \quad \mathbf{k}_{(+)} \cdot \mathbf{N}_{(+)}=0, \\
& \boldsymbol{\varphi}_{(+)} \cdot \mathbf{M}_{(+)}=0, \quad \mathbf{N}_{(+)} \cdot \boldsymbol{\psi}_{(+)}=0 .
\end{aligned}
$$


Аналогичные соотношения должны выполняться и на втором разрезе:

$$
\begin{array}{rr}
\mathcal{L}_{-}: \quad \mathbf{M}_{(-)} \cdot \mathbf{l}_{(-)}=0, & \mathbf{k}_{(-)} \cdot \mathbf{N}_{(-)}=0 \\
\boldsymbol{\varphi}_{(-)} \cdot \mathbf{M}_{(-)}=0, & \mathbf{N}_{(-)} \cdot \boldsymbol{\Psi}_{(-)}=0
\end{array}
$$

Таким образом, в окрестностях каждого из разрезов мы имеем два фундаментальных решения. Одно из них является определенным "глобально" нормированным фундаментальным решением $\boldsymbol{\Psi}$. Другое решение - локально определенное решение $\boldsymbol{\Psi}_{(+)}$, если речь идет о разрезе $\mathcal{L}_{+}$, или $\boldsymbol{\Psi}_{(-)}$, если речь идет о разрезе $\mathcal{L}_{-}$. Любые два фундаментальных решения в пересечении их областей определения связаны между собой некоторым линейным преобразованием, которое в нашем случае может зависеть от спектрального параметара:

$$
\boldsymbol{\Psi}(\xi, \eta, w)=\left\{\begin{array}{l}
\boldsymbol{\Psi}_{(+)}(\xi, \eta, w) \cdot \mathbf{C}_{+}(w) \\
\boldsymbol{\Psi}_{(-)}(\xi, \eta, w) \cdot \mathbf{C}_{-}(w)
\end{array}\right.
$$

Очевидно, что матрицы $\mathbf{C}_{ \pm}$, нормируюшие решения $\boldsymbol{\Psi}_{(+)}$и $\boldsymbol{\Psi}_{(-)}$, должны иметь вид $\mathbf{C}_{+}(w)=\Psi_{(+)}^{-1}\left(\xi_{0}, \eta_{0}, w\right)$ и $\mathbf{C}_{-}(w)=\Psi_{(-)}^{-1}\left(\xi_{0}, \eta_{0}, w\right)$. Эти выражения вместе с выражениями (26) позволяют получить аналогичное локальное выражение для $\Psi$ и $\Psi^{-1}$ и показать, что соответствующие фрагменты локальной структуры этих матричных функций удовлетворяют соотношениям, аналогичным (27) и (28). Сформулируем получаемые таким образом свойства в виде следуюшей теоремы, следствием которой будут явные выражения для скачков $\Psi_{\text {и }} \boldsymbol{\Psi}^{-1}$, которые они испытывают на разрезах $\mathcal{L}_{+}$и $\mathcal{L}_{-}$.

ТЕОрема 2. Для любого локального решения $\mathbf{U}, \mathbf{V}$ уравнений “нулевой кривизнье" (3) с условием (4) найдется такая окрестность $\Omega^{2}=\Omega_{\xi_{0}} \times \Omega_{\eta_{0}}$ начальной точки $P_{0}$, что:

1) нормированное фундаментальное решение $\boldsymbol{\Psi}(\xi, \eta, w)$ системы $(5)$ в областях $\Omega^{2} \times \Omega_{+}$и $\Omega^{2} \times \Omega_{-}\left(\right.$окрестностях разрезов $\mathcal{L}_{+}$и $\left.\mathcal{L}_{-}\right)$имеет локальную структуру

$$
\boldsymbol{\Psi}= \begin{cases}\lambda_{+}^{-1} e^{-i \sigma_{+}} \boldsymbol{\psi}_{+}(\xi, \eta, w) \otimes \mathbf{k}_{+}(w)+\mathbf{M}_{+}(\xi, \eta, w), & w \in \Omega_{+} \\ \lambda_{-}^{-1} e^{-i \sigma_{-}} \boldsymbol{\psi}_{-}(\xi, \eta, w) \otimes \mathbf{k}_{-}(w)+\mathbf{M}_{-}(\xi, \eta, w), & w \in \Omega_{-}\end{cases}
$$

и аналогичную локальную структуру имеет функция $\boldsymbol{\Psi}^{-1}(\xi, \eta, w)$ :

$$
\boldsymbol{\Psi}^{-1}= \begin{cases}\lambda_{+} e^{i \sigma_{+}} \mathbf{1}_{+}(w) \otimes \boldsymbol{\varphi}_{+}(\xi, \eta, w)+\mathbf{N}_{+}(\xi, \eta, w), & w \in \Omega_{+} \\ \lambda_{-} e^{i \sigma_{-} \mathbf{l}_{-}}(w) \otimes \boldsymbol{\varphi}_{-}(\xi, \eta, w)+\mathbf{N}_{-}(\xi, \eta, w), & w \in \Omega_{-}\end{cases}
$$

где вектор-строки (при $N=2,3)$ или $(d \times 2 d)$-матриць $($ при $N=2 d) \quad \mathbf{k}_{ \pm}(w)$, $\boldsymbol{\varphi}_{ \pm}(\xi, \eta, w)$, вектор-столбив $($ при $N=2,3)$ или $(2 d \times d)$-матрицьи $($ при $N=2 d)$ $\mathbf{l}_{ \pm}(w), \boldsymbol{\psi}_{ \pm}(\xi, \eta, w)$, а также матрицы $\mathbf{M}_{ \pm}(\xi, \eta, w), \mathbf{N}_{ \pm}(\xi, \eta, w)$ голоморфньи в окрестностях $\mathcal{L}_{+}$или $\mathcal{L}_{-}$, соответственно, а функиии $\lambda_{ \pm}$и $\sigma_{ \pm}$определены в $(20) ;$ знак $\otimes n р и ~ N=2,3$ означает тензорное произведение, а при $N=2 d$ долэсен пониматься как обичное матричное умножсение; 
2) в окрестностях разрезов $\mathcal{L}_{+}$и $\mathcal{L}_{-}$выполнены соотношения

$$
\begin{array}{lllll}
\Omega^{2} \times \Omega_{+}: & \mathbf{M}_{+} \cdot \mathbf{l}_{+}=0, & \mathbf{k}_{+} \cdot \mathbf{N}_{+}=0, & \boldsymbol{\varphi}_{+} \cdot \mathbf{M}_{+}=0, & \mathbf{N}_{+} \cdot \boldsymbol{\psi}_{+}=0 \\
\Omega^{2} \times \Omega_{-}: & \mathbf{M}_{-} \cdot \mathbf{l}_{-}=0, & \mathbf{k}_{-} \cdot \mathbf{N}_{-}=0, & \boldsymbol{\varphi} \mathbf{M}_{-}=0, & \mathbf{N}_{-} \cdot \boldsymbol{\psi}_{-}=0
\end{array}
$$

3) в начальной точке $P_{0}\left(\xi_{0}, \eta_{0}\right)$ значения функиий $\varphi_{ \pm}, \boldsymbol{\psi}_{ \pm}$им матрии $\mathbf{M}_{ \pm} u \mathbf{N}_{ \pm}$ выражаются через компоненты функиий $\mathbf{k}_{ \pm} u \mathbf{l}_{ \pm}$:

$$
\begin{array}{ll}
\boldsymbol{\psi}_{0 \pm}(w)=\mathbf{l}_{ \pm}(w) \cdot\left(\mathbf{k}_{ \pm}(w) \cdot \mathbf{l}_{ \pm}(w)\right)^{-1}, & \mathbf{M}_{0 \pm}=\mathbf{I}-\boldsymbol{\psi}_{0 \pm}(w) \otimes \mathbf{k}_{ \pm}(w) \\
\boldsymbol{\varphi}_{0 \pm}(w)=\left(\mathbf{k}_{ \pm}(w) \cdot \mathbf{l}_{ \pm}(w)\right)^{-1} \cdot \mathbf{k}_{ \pm}(w), & \mathbf{N}_{0 \pm}=\mathbf{I}-\mathbf{l}_{ \pm}(w) \otimes \boldsymbol{\varphi}_{0 \pm}(w)
\end{array}
$$

где во всех выражсениях справа и слева выбираются одновременно только верхние или только нижние знаки.

Для функции, испытываюшей скачок на разрезе $L$, обозначим квадратными и фигурными скобками величины ее скачка и "непрерывной" части в точке $\tau \in L$, определяемые соответственно как полуразность и полусумма ее предельных значений слева и справа от разреза в этой точке. Тогда на основании определений (20)

$$
\begin{aligned}
& \left\{\lambda_{+}\right\}_{\tau_{+}}=0, \quad\left\{\lambda_{+}^{-1}\right\}_{\tau_{+}}=0, \quad\left[\sigma_{+}\right]_{\tau_{+}}=-i \frac{\pi}{2} \mu_{+}\left(\tau_{+}\right), \quad\left\{\sigma_{+}\right\}_{\tau_{+}}=-\frac{1}{2} \oint_{L_{+}} \frac{\mu_{+}\left(\zeta_{+}\right) d \zeta_{+}}{\zeta_{+}-\tau_{+}} \\
& \left\{\lambda_{-}\right\}_{\tau_{-}}=0, \quad\left\{\lambda_{-}^{-1}\right\}_{\tau_{-}}=0, \quad\left[\sigma_{-}\right]_{\tau_{-}}=-i \frac{\pi}{2} \mu_{-}\left(\tau_{-}\right), \quad\left\{\sigma_{-}\right\}_{\tau_{-}}=-\frac{1}{2} \oint_{L_{-}} \frac{\mu_{-}\left(\zeta_{-}\right) d \zeta_{-}}{\zeta_{-}-\tau_{-}}
\end{aligned}
$$

где $\tau_{+} \in L_{+}, \tau_{-} \in L_{-}$, а интегралы понимаются в смысле главного значения по Коши. Для скачков и непрерывных частей произведений этих функций в точках $\tau_{+} \in L_{+}$или $\tau_{-} \in L_{-}$удобно использовать более явные выражения вида

$$
\begin{aligned}
{\left[\lambda e^{i \sigma}\right] } & =[\lambda] e^{i\{\sigma\}} \cos [\sigma], & {\left[\lambda^{-1} e^{-i \sigma}\right] } & =\left[\lambda^{-1}\right] e^{-i\{\sigma\}} \cos [\sigma], \\
\left\{\lambda e^{i \sigma}\right\} & =i[\lambda] e^{i\{\sigma\}} \sin [\sigma], & \left\{\lambda^{-1} e^{-i \sigma}\right\} & =-i\left[\lambda^{-1}\right] e^{-i\{\sigma\}} \sin [\sigma] .
\end{aligned}
$$

Используя эти соотношения, из теоремы 2 получаем

СЛЕДСТВИЕ 2. СКачки $\boldsymbol{\Psi} и \boldsymbol{\Psi}^{-1}$ на разрезах $L_{ \pm}$имеют вид

$$
[\boldsymbol{\Psi}]_{L_{ \pm}}=\left[\lambda_{ \pm}^{-1}\right] e^{-i\left\{\sigma_{ \pm}\right\}} \cos \left[\sigma_{ \pm}\right] \boldsymbol{\psi}_{ \pm} \otimes \mathbf{k}_{ \pm}, \quad[\boldsymbol{\Psi}]_{L_{ \pm}}^{-1}=\left[\lambda_{ \pm}\right] e^{i\left\{\sigma_{ \pm}\right\}} \cos \left[\sigma_{ \pm}\right] \mathbf{l}_{ \pm} \otimes \boldsymbol{\varphi}_{ \pm}
$$

тогда как аналогичные выражения для непрерывных частей этих функиий позволяют представить матричные функиии $\mathbf{M}_{ \pm} u \mathbf{N}_{ \pm}$в точках разрезов $\mathcal{L}_{ \pm}$в виде

$$
\begin{aligned}
& \mathbf{M}_{ \pm}=\{\boldsymbol{\Psi}\}_{L_{ \pm}}+i\left[\lambda_{ \pm}^{-1}\right] e^{-i\left\{\sigma_{ \pm}\right\}} \sin \left[\sigma_{ \pm}\right] \boldsymbol{\psi}_{ \pm} \otimes \mathbf{k}_{ \pm} \\
& \mathbf{N}_{ \pm}=\left\{\boldsymbol{\Psi}^{-1}\right\}_{L_{ \pm}}-i\left[\lambda_{ \pm}\right] e^{i\left\{\sigma_{ \pm}\right\}} \sin \left[\sigma_{ \pm}\right] \mathbf{1}_{ \pm} \otimes \boldsymbol{\varphi}_{ \pm}
\end{aligned}
$$

где справа и слева выбираются одновременно только верхние или нижние знаки, а скачки и непрерывные части $\lambda_{+}, \sigma_{+} u \lambda_{-}, \sigma_{-}$вычисляются на разрезах $L_{+} u$ $L_{-}$, соответственно. 


\section{7. “КООР ДИНАТЫ" В ПРОСТРАНСТВАХ ЛОКАЛЬНЫХ РЕШЕНИЙ}

7.1. Независимые функциональные параметры. Среди фрагментов локальной структуры $\boldsymbol{\Psi}$ и $\boldsymbol{\Psi}^{-1}$ присутствуют четыре векторные (при $\left.N=2,3\right)$ или $(d \times 2 d)$ и $(2 d \times d)$-матричные (при $N=2 d)$ функции, зависящие только от спектрального параметра - функции $\mathbf{k}_{+}(w), \mathbf{l}_{+}(w)$, голоморфные в области $\Omega_{+}$, и функции $\mathbf{k}_{-}(w), \mathbf{l}_{-}(w)$, голоморфные в области $\Omega_{-}$. Из выражений $(29),(30)$ нетрудно увидеть, что эти функции определены на самом деле лишш в "проективном" смысле, т.е. в векторном случае $(N=2,3)$ с точностью до умножения на произвольную скалярную (голоморфную в окрестности соответствуюшего разреза) функцию от $w$ и одновременного деления другого сомножителя на эту же функцию. В матричном случае $(N=2 d)$ этот множитель есть произвольная невырожденная $(d \times d)$-матричная функция спектрального параметра. Этот произвол можно фиксировать, положив при $N=2,3$ первые компоненты векторов $\mathbf{k}_{ \pm}(w)$ и $\mathbf{l}_{ \pm}(w)$ равными 1, а при $N=2 d$ - первый $d$-мерный блок этих матриц равным единичной матрище:

$$
\begin{aligned}
\mathbf{k}_{ \pm}^{N=2}(w) & =\left\{1, \mathbf{u}_{ \pm}(w)\right\}, & \mathbf{l}_{ \pm}^{N=2}(w) & =\left\{1, \mathbf{p}_{ \pm}(w)\right\}^{\mathrm{T}}, \\
\mathbf{k}_{ \pm}^{N=3}(w) & =\left\{1, \mathbf{u}_{ \pm}(w), \mathbf{v}_{ \pm}(w)\right\}, & \mathbf{l}_{ \pm}^{N=3}(w) & =\left\{1, \mathbf{p}_{ \pm}(w), \mathbf{q}_{ \pm}(w)\right\}^{\mathrm{T}}, \\
\mathbf{k}_{ \pm}^{N=2 d}(w) & =\left\{I_{d}, \mathbf{u}_{ \pm}(w)\right\}, & \mathbf{l}_{ \pm}^{N=2 d}(w) & =\left\{I_{d}, \mathbf{p}_{ \pm}(w)\right\}^{\mathrm{T}},
\end{aligned}
$$

где в последнем случае $\mathbf{u}_{ \pm}$и $\mathbf{p}_{ \pm}$есть $(d \times d)$-матричные функции. Компоненты $\mathbf{k}_{ \pm}(w)$ и $\mathbf{l}_{ \pm}(w)$, к которым в случаях $N=2,3$ могут быть добавлены функции $\mu_{ \pm}(w)$, образуют, как мы увидим далее, полньй набор независимых функциональных параметров, которые могут играть роль "координат" в пространстве локальных решений уравнений "нулевой кривизны" (3) с условиями (4).

7.2. Данные монодромии как функциональные параметры. Благодаря наличию выражений $(31)$, векторные (или матричные) функции $\mathbf{k}_{ \pm}(w)$ и $\mathbf{l}_{ \pm}(w)$ имеют весьма простую интерпретацию как величины, определяюшие начальные данные (в упомянутом выше "проективном" смысле) для ветвяшейся на данном разрезе части линейно независимых решений, образуюших нормированную фундаментальную систему решений для $\boldsymbol{\Psi}$ и $\boldsymbol{\Psi}^{-1}$. Независимые компоненты этих функций можно и иначе, более ясно, интерпретировать как полньй набор данных, определяюших монодромию нормированного фундаментального решения $\boldsymbol{\Psi}$ на спектральной плоскости. Для этого рассмотрим на спектральной плоскости два пути $t_{+}$и $t_{-}$, ведушие, скажем для определенности, с левого берега разреза $L_{+}$или $L_{-}$на его правый берег по часовой стрелке в обход точек $w=\xi$ или $w=\eta$, соответственно. В результате аналитического продолжения вдоль каждого из этих путей нормированное фундаментальное решение $\boldsymbol{\Psi}$ системы (5) переходит в другое решение $\widetilde{\boldsymbol{\Psi}}$, отличаюшееся от $\boldsymbol{\Psi}$ некоторым линейным преобразованием $\boldsymbol{\Psi} \stackrel{t_{ \pm}}{\longrightarrow} \widetilde{\boldsymbol{\Psi}}=\boldsymbol{\Psi} \mathbf{T}_{ \pm}(w)$. Матрицы этих преобразований (матрицы монодромии) вычисляются по формуле $\mathbf{T}_{ \pm}(w)=\Psi^{-1} \cdot \widetilde{\Psi}$, если воспользоваться локальными представления- 
ми (29), (30) для $\boldsymbol{\Psi}_{\text {и }} \boldsymbol{\Psi}^{-1}$ в окрестности разрезов $L_{ \pm}$:

$$
\begin{aligned}
& \mathbf{T}_{+}(w)=\mathbf{I}-\left(1+e^{-\pi \mu_{+}(w)}\right) \mathbf{l}_{+}(w) \otimes\left(\mathbf{k}_{+}(w) \cdot \mathbf{l}_{+}(w)\right)^{-1} \otimes \mathbf{k}_{+}(w) \\
& \mathbf{T}_{-}(w)=\mathbf{I}-\left(1+e^{-\pi \mu_{-}(w)}\right) \mathbf{l}_{-}(w) \otimes\left(\mathbf{k}_{-}(w) \cdot \mathbf{l}_{-}(w)\right)^{-1} \otimes \mathbf{k}_{-}(w)
\end{aligned}
$$

Как видно из этих выражений, матрицы монодромии $\mathbf{T}_{ \pm}(w)$ полностью определяются компонентами векторов (матриц) $\mathbf{k}_{ \pm}(w), \mathbf{l}_{ \pm}(w)$ и функциями $\mu_{ \pm}(w)$, задающими спинорное поле. Верно и обратное: все независимые функциональные параметры, содержащиеся в компонентах $(32)$ векторов $\mathbf{k}_{ \pm}(w), \mathbf{l}_{ \pm}(w)$, и функции $\mu_{+}(w)$ и $\mu_{-}(w)$ однозначно определяются по компонентам матриц монодромии (33). Как следует из теорем 1 и 2 , набор функциональных параметров вида (32) вместе с функциями $\mu_{+}(w)$ и $\mu_{-}(w)$, называемый далее "расширенными данными монодромии", может быть однозначно определен для любого локального решения уравнений “нулевой кривизны” (3) с условиями (4).

Определенные таким образом (расширенные) данные монодромии могут играть роль, аналогичную данным рассеяния в методе обратной задачи рассеяния, поэтому переход к таким "координатам" в пространстве решений аналогично “преобразованию рассеяния" будем называть “преобразованием монодромии”.

\section{8. ОБРАТНАЯ ЗАДАЧА ПРЕОБРАЗОВАНИЯ МОНОДРОМИИ}

Покажем теперь, что для произвольно выбираемых функций (32), зависящих только от спектрального параметра и голоморфных в некоторой окрестности точек спектральной плоскости $w=\xi_{0}$ (для функций с индексом “+”) и $w=\eta_{0}$ (для функций с индексом “-”), всегда существует, и притом единственное, локальное решение уравнений "нулевой кривизны" (3) с условиями (4), для которого нормированное фундаментальное решение линейной системы (5) характеризуется этими (расширенными) данными монодромии.

8.1. Определяющая система линейных сингулярных интегральных уравнений. Введем дополнительные обозначения. Параметры $\tau, \zeta$, пробегаюшие разрезы $L_{+}$и $L_{-}$, снабдим соответствующими индексами + или,- а для пар функций, определяемых на $L_{ \pm}$и отличающихся индексами + или - , введем соответствующие функции без индексов, например

$$
\mathbf{k}(\tau)=\left\{\begin{array}{ll}
\mathbf{k}_{+}(\tau), & \tau=\tau_{+} \in L_{+}, \\
\mathbf{k}_{-}(\tau), & \tau=\tau_{-} \in L_{-},
\end{array} \quad \boldsymbol{\varphi}(\xi, \eta, \tau)= \begin{cases}\boldsymbol{\varphi}_{+}(\xi, \eta, \tau), & \tau=\tau_{+} \in L_{+} \\
\boldsymbol{\varphi}_{-}(\xi, \eta, \tau), & \tau=\tau_{-} \in L_{-}\end{cases}\right.
$$

Аналогично определяются и функции $\lambda, \sigma, \mathbf{1}, \boldsymbol{\psi}, \mathbf{M}$ и $\mathbf{N}$.

Используя формулы Сохоцкого-Племеля и выражения (24), непрерывные на $L$ части $\boldsymbol{\Psi}$ и $\boldsymbol{\Psi}^{-1}$ можно представить в виде

$$
\{\boldsymbol{\Psi}\}_{\tau}=\mathbf{I}+\frac{1}{i \pi} \oint_{L} \frac{[\boldsymbol{\Psi}]_{\zeta}}{\zeta-\tau} d \zeta, \quad\left\{\boldsymbol{\Psi}^{-1}\right\}_{\tau}=\mathbf{I}+\frac{1}{i \pi} \oint_{L} \frac{\left[\boldsymbol{\Psi}^{-1}\right]_{\zeta}}{\zeta-\tau} d \zeta
$$


где интегралы по контуру $L=L_{+} \cup L_{-}$понимаются в смысле главного значения по Коши, а переменная $\tau=\tau_{+} \in L_{+}$или $\tau=\tau_{-} \in L_{-}$. Подстановка этих выражений, а также соотношений, приведенных в следствии 2 , в алгебраические связи $\mathbf{M} \cdot \mathbf{1}=0$ и $\mathbf{k} \cdot \mathbf{N}=0$, установленные в теореме 2 , приводит к следующей теореме.

ТЕОРема 3. Для любого локального решения уравнений нулевой кривизны (3) с условиями (4) фрагменты алгебраической структурь матричных функиий $\Psi$ и $\Psi^{-1}$ на разрезах $L_{+}$и $L_{-}$должны удовлетворять интегральным уравнениям

$$
\begin{aligned}
& \nu(\xi, \eta, \tau) \cdot \boldsymbol{\varphi}(\xi, \eta, \tau)+\frac{1}{\pi i} \oint_{L} \frac{\mathcal{K}(\xi, \eta, \tau, \zeta)}{\zeta-\tau} \cdot \boldsymbol{\varphi}(\xi, \eta, \zeta) d \zeta=\mathbf{k}(\tau), \\
& \boldsymbol{\psi}(\xi, \eta, \tau) \cdot \widetilde{\nu}(\xi, \eta, \tau)+\frac{1}{\pi i} \oint_{L} \boldsymbol{\psi}(\xi, \eta, \zeta) \cdot \frac{\widetilde{\mathcal{K}}(\xi, \eta, \tau, \zeta)}{\zeta-\tau} d \zeta=\mathbf{l}(\tau),
\end{aligned}
$$

где интеграль по $L=L_{+} \cup L_{-}$понимаются в смысле главного значения по Коши, точка в матричном случае $(N=2 d)$ означает матричное произведение, а скалярные $($ при $N=2,3)$ или $(d \times d)$-матричные $($ при $N=2 d)$ ядра и коэффициенты имеют вид

$$
\begin{array}{llrl}
\mathcal{K}(\xi, \eta, \tau, \zeta) & =-\left[\lambda e^{i \sigma}\right]_{\zeta} \mathcal{H}(\tau, \zeta), & \nu(\xi, \eta, \tau) & =\left\{\lambda e^{i \sigma}\right\}_{\tau} \mathcal{H}(\tau, \tau), \\
\widetilde{\mathcal{K}}(\xi, \eta, \tau, \zeta) & =-\left[\lambda^{-1} e^{-i \sigma}\right]_{\zeta} \mathcal{H}(\zeta, \tau), & \widetilde{\nu}(\xi, \eta, \tau) & =\left\{\lambda^{-1} e^{-i \sigma}\right\}_{\tau} \mathcal{H}(\tau, \tau),
\end{array}
$$

где $\mathcal{H}(x, y) \equiv(\mathbf{k}(x) \cdot \mathbf{l}(y))$, а каждый из параметров $\zeta$ и $\tau$ пробегает контур $L=L_{+} \cup$ $L_{-}$.

Поясним здесь, что каждый из интегралов в (35) представляет собой сумму двух интегралов по разрезам $L_{+}$и $L_{-}$, а каждое из интегральных соотношений (35) в более детальной записи представляет собой не одно, а два интегральных уравнения, получаемых из исходного поочередной подстановкой в него $\tau=\tau_{+}$и $\tau=\tau_{-}$, так что в получающихся двух парах уравнений будет не два, а четыре различных ядра, определяемых функциями (или $(d \times d)$-матрицами при $N=2 d)$ вида $\mathcal{H}_{ \pm \pm}(\tau, \zeta) \equiv \mathcal{H}\left(\tau_{ \pm}, \zeta_{ \pm}\right)$.

8.2. Сушествование и единственность решения обратной задачи. Вьше было показано, что для любого локального решения уравнений нулевой кривизны (3) с условиями (4) должны с необходимостью выполняться соотношения (35). Однако (35) можно рассматривать как линейные сингулярные интегральные уравнения для определения компонент величин $\varphi$ и $\psi$ по расширенным данным монодромии - компонентам $(32)$ векторов (матриц) $\mathbf{k}$ и $\mathbf{l}$ и спинорным переменным $\mu_{+}(w)$ и $\mu_{-}(w)$. При этом легко заметить, что эти уравнения не образуют связанной системы и, более того, в скалярном случае каждое из них распадается на отдельные уравнения для каждой компоненты искомых функций или, при $N=2 d$, для каждого из $(d \times d)$-блоков, образующих $(d \times 2 d)$ и $(2 d \times d)$-матрицы $\varphi$ и $\boldsymbol{\psi}$

Специфика получаемых линейных сингулярных интегральных уравнений состоит в структуре контура интегрирования, образованного двумя незамкнутыми и непересекаюшимися кривыми. Другим важным условием, вытекаюшим из условий спектральной 
задачи, является то, что решения $\varphi$ и $\psi$ должны быть ограниченными на концах каждой из двух составляюших контур кривых. На основании обшей теории линейных сингулярных интегральных уравнений [31], [32] можно показать, что соответствующие однородные характеристические уравнения, отвечающие каждому из полученных независимых скалярных или $(d \times d)$-матричных интегральных уравнений, на которые распадается система (35), оказываются имеюшими нулевой индекс ${ }^{8)}$. Поэтому соответствуюшие полные (т.е. с регулярной частью) интегральные уравнения допускают регуляризацию, которая превращает их в эквивалентные квазифредгольмовы линейные интегральные уравнения 2-го рода. Для построения его решения можно использовать обычный метод последовательных приближений и доказать затем существование и единственность решения, следуя стандартной схеме. При этом существенно используется то, что мы рассматриваем только локальные решения, а именно, при произвольном выборе данных монодромии всегда найдется достаточно малая окрестность $\Omega_{\xi_{0}} \times \Omega_{\eta_{0}}$ начальной точки $P_{0}\left(\xi_{0}, \eta_{0}\right)$, в которой соответствуюший интегральный оператор будет сжимающим, и сходимость последовательных приближений можно доказать мажорантным методом. Подробное доказательство выходит, очевидно, за рамки настоящей работы, поэтому мы сформулируем здесь лиш окончательный результат.

ТЕОрема 4. Для произвольно выбираемых расширенных данных монодромии, состоящих из двух скалярных $\mu_{+}(w), \mu_{-}(w)$ и двух пар векторных $($ при $N=2,3)$ или только из двух пар $(d \times 2 d)-u(2 d \times d)$-матричных $(\text { при } N=2 d)^{9)}$ функций $\mathbf{k}_{+}(w), \mathbf{l}_{+}(w) u \mathbf{k}_{-}(w), \mathbf{1}_{-}(w)$, голоморфных соответственно в некоторых окрестностях $\Omega_{+} u \Omega_{-}$точек $w=\xi_{0} u w=\eta_{0}$ спектральной плоскости, найдется такая окрестность $\Omega^{2}=\Omega_{\xi_{0}} \times \Omega_{\eta_{0}}$ начальной точки $P_{0}\left(\xi_{0}, \eta_{0}\right)$, в которой каждое из уравнений (35) с коэффициентами, определенными в (20) и (36) с учетом (34), имеет, и притом единственное, решение $\boldsymbol{\varphi}_{ \pm}(\xi, \eta, w) u \boldsymbol{\psi}_{ \pm}(\xi, \eta, w)$, соответственно. Построенные по этим решениям матричные функиии

$$
\begin{aligned}
& \boldsymbol{\Psi}=\mathbf{I}+\frac{1}{i \pi} \int_{L} \frac{\left[\lambda^{-1} e^{-i \sigma}\right]_{\zeta}}{\zeta-w} \boldsymbol{\psi}(\xi, \eta, \zeta) \otimes \mathbf{k}(\zeta) d \zeta \\
& \widetilde{\boldsymbol{\Psi}}=\mathbf{I}+\frac{1}{i \pi} \int_{L} \frac{\left[\lambda e^{i \sigma}\right]_{\zeta}}{\zeta-w} \mathbf{l}(\zeta) \otimes \boldsymbol{\varphi}(\xi, \eta, \zeta) d \zeta
\end{aligned}
$$

обладают следующими свойствами:

1) они являются взаимно обратныцми: $\widetilde{\Psi}=\Psi^{-1}$;

2) $\Psi$ есть фундаментальное решение системы (5) с условиями (4) и нормировкой (14).

\footnotetext{
8) При вычислении индекса необходимо учитывать особенности на концах контура интегрирования, обусловленные присутствием скачков $\lambda$ в подинтегральных выражениях.

9) Напомним, что при $N=2 d$ и $d>1$ спинорные поля не рассматриваются, т.е. в этом случае $\mu_{+}=0$ и $\mu_{-}=0$.
} 
8.3. Вычисление решений уравнений "нулевой кривизны" по расширенным данным монодромии. Задавшись некоторым расширенным набором данных монодромии (32) и найдя отвечающее этим данным решение какого-либо из интегральных уравнений (35), локальное решение уравнений “нулевой кривизны” (3) с условиями (4) можно вычислить, воспользовавшись тем, что это решение определяется согласно системе (5) по разложению $\boldsymbol{\Psi}=\mathbf{I}+(1 / w) \mathbf{R}+\cdots$ при $w \rightarrow \infty$ в виде

$$
\mathbf{U}=2 i \partial_{\xi} \mathbf{R}, \quad \mathbf{V}=2 i \partial_{\eta} \mathbf{R},
$$

где матрица $\mathbf{R}(\xi, \eta)$ в силу (37) представляется выражениями

$$
\mathbf{R}=-\frac{1}{i \pi} \int_{L}\left[\lambda^{-1} e^{-i \sigma}\right]_{\zeta} \boldsymbol{\psi}(\xi, \eta, \zeta) \otimes \mathbf{k}(\zeta) d \zeta=\frac{1}{i \pi} \int_{L}\left[\lambda e^{i \sigma}\right]_{\zeta} \mathbf{l}(\zeta) \otimes \boldsymbol{\varphi}(\xi, \eta, \zeta) d \zeta
$$

и, следовательно, вычисляется в квадратурах по данным монодромии и соответствующему решению любого из интегральных уравнений (35).

\section{9. ПРОСТРАНСТВА ЛОКАЛЬНЫХ РЕШЕНИЙ ИНТЕГРИРУЕМЫХ РЕДУКЦИЙ УРАВНЕНИЙ ЭЙНШТЕЙНА}

Приведенная выше конструкция позволила выразить обшее локальное решение уравнений "нулевой кривизны" (3) с жордановыми условиями (4) в квадратурах через данные монодромии и соответствуюшее им решение построенной нами линейной системы сингулярных интегральных уравнений. Теперь нетрудно показать, как эта конструкция должна быть модифищирована для получения аналогичного представления общего локального решения для каждой из рассматриваемых нами интегрируемых редукций уравнений Эйнштейна.

9.1. Условия существования матричных интегралов $\mathbf{K}(w)$ и $\mathbf{L}(w)$. Дополнительные условия, сужаюшие пространство локальных решений уравнений "нулевой кривизны" с жордановыми условиями до пространства решений обобщенных уравнений Эрнста, сводятся, очевидно, к требованию существования у линейных систем (5) во всех случаях $(N=2,3,2 d)$ эрмитовых матричных интегралов $\mathbf{K}(w)$ вида $(8)$, (9), а при $N=2 d$ - еше и антисимметричного матричного интеграла $\mathbf{L}(w)$ вида $(10)$, которые приобретают с учетом нормировки (14), (13) вид $(15)^{10)}$.

Из голоморфности $\Psi$ и $\Psi^{-1}$ вне разреза следует, что условия (15) достаточно было бы удовлетворить на самом разрезе. Используя в выражениях (15) для этих интегралов локальные представления (29), (30) для $\boldsymbol{\Psi}$ и $\boldsymbol{\Psi}^{-1}$ на разрезах, можно показать, что эти условия сводятся к наложению некоторых связей между расширенными данными монодромии (32). Эти связи легко разрешаются. В матричном случае $(N=2 d)$ условие

\footnotetext{
${ }^{10)}$ Напомним здесь, что в матричном случае $N=2 d$ мы ограничились рассмотрением моделей с симметричными $(d \times d)$-матричными потенциалами Эрнста. Для моделей, в которых потенциалы Эрнста представляют собой эрмитовы $(d \times d)$-матрицы, интеграл $\mathbf{K}(w)$ должен быть симметричным, а интеграл $\mathbf{L}(w)$ - эрмитовым [17].
} 
сушествования антисимметричного интеграла $\mathbf{L}(w)$ приводит к тому, что матрищы $\mathbf{u}_{ \pm}$ и $\mathbf{p}_{ \pm}$должны быть симметричными. Условие существования эрмитова интеграла $\mathbf{K}(w)$ приводит к явному выражению компонент $\mathbf{l}(w)$ через эрмитово сопряженные величины $\mathbf{k}^{\dagger}(w)^{11)}$ :

$$
\mathbf{l}(w)=S_{0}^{2}(w) \mathbf{W}_{0}^{-1}(w) \cdot \mathbf{k}^{\dagger}(w),
$$

где скалярный множитель $S_{0}^{2}(w)=\left(w-\xi_{0}\right)\left(w-\eta_{0}\right)$ необходим, чтобы компенсировать нули детерминанта матрицы $\mathbf{W}_{0}(w)$ и сделать компоненты получаемых векторов $\mathbf{l}_{+}(w)$ и $\mathbf{l}_{-}(w)$ голоморфными на разрезах $L_{+}$и $L_{-}$, соответственно.

9.2. Данные монодромии для решений редуцированных уравнений Эйнштейна. В силу выражений (40) независимыми функциональными параметрами, которые могут служить “координатами” в пространствах локальных решений редуцированных уравнений Эйнштейна, являются только присутствующие в компонентах векторов (с учетом обозначений (34) и сноски к (40))

$$
\begin{aligned}
& \mathbf{k}^{N=2}(w)=\{1, \mathbf{u}(w)\}, \\
& \mathbf{k}^{N=3}(w)=\{1, \mathbf{u}(w), \mathbf{v}(w)\},
\end{aligned} \quad\left\{\begin{array}{l}
\mathbf{k}^{N=2 d}(w)=\left\{I_{d}, \mathbf{u}(w)\right\} \\
\mathbf{u}^{\mathrm{T}}(w)=\mathbf{u}(w)
\end{array}\right.
$$

скалярные функции спектрального параметра $\mathbf{u}_{ \pm}(w)$ для гравитационных полей в вакууме $(N=2)$, дополненные функциями $\mathbf{v}_{ \pm}(w)$, отвечаюшими за наличие электромагнитных полей $(N=3)$, и функциями $\mu_{+}(w)$ и $\mu_{-}(w)$, задающими безмассовое спинорное поле $(N=2,3)$, или $(d \times d)$-матричные функции $\mathbf{u}_{ \pm}(w)$ для рассматриваемых моделей струнной гравитации $(N=2 d)$. В матричном случае $(N=2 d)$ матрицы $\mathbf{u}_{ \pm}(w)$ должны быть симметричными для моделей с симметричными потенциалами Эрнста ${ }^{12)}$. Если вместо расширенных данных монодромии (32) мы выбираем данные монодромии в виде выражений (40), (41), то пространство локальных решений уравнений "нулевой кривизны" сужается до пространства всех локальных решений соответствующих редуцированных уравнений Эйнштейна.

9.3. Вычисление решений обобшенных уравнений Эрнста по данным монодромии. Аналогично рассмотренному выше построению решений для уравнений "нулевой кривизны" сначала мы должны, задавшись данными монодромии вида (40),

11) Здесь необходимо учитывать, что ввиду различной структуры разрезов в гиперболическом $(\epsilon=1)$ и эллиптическом $(\epsilon=-1)$ случаях определенное ранее эрмитово сопряжение действует даже на скалярные функции спектрального параметра по-разному:

$$
\epsilon=1: \quad \mathbf{u}^{\dagger}(w)=\left\{\begin{array}{ll}
\overline{\mathbf{u}_{+}^{\mathrm{T}}(\bar{w})}, & w \in \Omega_{+}, \\
\overline{\mathbf{u}_{-}^{\mathrm{T}}(\bar{w})}, & w \in \Omega_{-},
\end{array} \quad \epsilon=-1: \quad \mathbf{u}^{\dagger}(w)= \begin{cases}\overline{\mathbf{u}_{-}^{\mathrm{T}}(\bar{w})}, & w \in \Omega_{+}, \\
\overline{\mathbf{u}_{+}^{\mathrm{T}}(\bar{w})}, & w \in \Omega_{-} .\end{cases}\right.
$$

12) Для моделей с эрмитовыми потенциалами Эрнста эрмитово сопряжение ${ }^{\dagger}$ в $(40)$ должно быть заменено на транспонирование, а матричные функции $\mathbf{u}_{ \pm}(w)$ в (41) вместо условия симметричности должны удовлетворять соотношению $\mathbf{u}^{\dagger}(w)=-\mathbf{u}(w)$. 
(41), решить одно из определяюших интегральных уравнений (35). По этому решению вычисляем в квадратурах компоненты матрицы $\mathbf{R}$ по формулам (39), а соответствующие выражения для $\mathbf{U}$ и $\mathbf{V}$ - по формулам (38). Скалярньй потеннциал Эрнста $\mathcal{E}$ при $N=2$, или $\mathcal{E}, \Phi$ при $N=3$, или $(d \times d)$-матрица $\mathcal{E}$ в случае $N=2 d$, определяемые, как известно, выражениями $\partial_{\xi} \mathcal{E}=-\mathbf{U}_{(1)}{ }^{(2)}, \partial_{\eta} \mathcal{E}=-\mathbf{V}_{(1)}{ }^{(2)}$ и $\partial_{\xi} \Phi=\mathbf{U}_{(1)}^{(3)}$, $\partial_{\eta} \Phi=\mathbf{V}_{(1)}^{(3)}$, могут быть вычислены по формулам $(d \geqslant 1)$

$$
N=2 d: \quad \mathcal{E}=\mathcal{E}_{0}-2 i \mathbf{R}_{(1)}^{(2)}, \quad N=3: \quad \mathcal{E}=\mathcal{E}_{0}-2 i \mathbf{R}_{(1)}{ }^{(2)}, \quad \Phi=2 i \mathbf{R}_{(1)}{ }^{(3)},
$$

где $\mathcal{E}_{0}$ есть значение потенциала Эрнста в начальной точке, определенное условиями нормировки, индексы в скобках при $N=2,3$ означают номера компонент матриш, а при $N=2 d$ и $d>1$ выражение $(\ldots)_{(1)}^{(2)}$ есть верхний правый $(d \times d)$-блок $(2 d \times 2 d)$-матрицы.

Таким образом, описанное решение прямой и обратной задач преобразования монодромии устанавливает взаимно однозначное соответствие между пространством локальных решений уравнений "нулевой кривизны" и пространством произвольно выбираемых расширенных данных монодромии (32) или редуцированных уравнений Эйнштейна и данных монодромии (40), (41). Говоря условно, данные монодромии действительно могут играть роль "координат" в бесконечномерном пространстве локальных решений интегрируемых редукций уравнений Эйнштейна. Устанавливаюшая это соответствие определяющая система линейных сингулярных интегральных уравнений заменяет собой динамические уравнения для полей и может эффективно использоваться для интегрирования рассматривавшихся нами уравнений Эйнштейна при наличии пространственно-временных симметрий.

Благодарности. Автор выражает благодарность А. К. Погребкову и А. Б. Шабату за полезные обсуждение и критику. Работа выполнена при частичной поддержке РФФИ (гранты № № 02-01-00729, 02-02-17372) и программ “Нелинейная динамика” РАН и "Ведушие научные школы РФ" (грант НШ-1697.2003.1).

\section{Список литературы}

[1] В. А. Белинский, В. Е. Захаров. ЖЭТФ. 1978. Т. 75. С. 1953; 1979. Т. 77. С. 3.

[2] B. K. Harrison. Phys. Rev. Lett. 1978. V. 41. P. 1197.

[3] G. Neugebauer. J. Phys. A. 1979. V. 12. P. L67; 1980. V. 13. P. 1737.

[4] I. Hauser, F. J. Ernst. Phys. Rev. D. 1979. V. 20. P. 362.

[5] W. Kinnersley. J. Math. Phys. 1977. V. 18. P. 1529.

[6] W. Kinnersley, D. M. Chitre. J. Math. Phys. 1977. V. 18. P. 1538.

[7] W. Kinnersley, D. M. Chitre. J. Math. Phys. 1978. V. 19. P. 1926.

[8] I. Hauser, F. J. Ernst. Phys. Rev. D. 1979. V. 20. P. 1783.

[9] Г. А. Алексеев. Письма в ЖЭТФ. 1980. Т. 32. С. 301.

[10] Г. А. Алексеев. ДАН СССР. 1983. Т. 268. № 6. С. 1347.

[11] В. А. Белинский. ЖЭТФ. 1979. Т. 77. С. 1239.

[12] В. А. Белинский. Письма в ЖЭТФ. 1979. Т. 30. С. 32.

[13] I. Bakas. Nucl. Phys. B. 1994. V. 428. P. 374; hep-th/9402016. 
[14] A. Kumar, K. Ray. Phys. Lett. B. 1995. V. 358. P. 223.

[15] D. V. Gal'tsov. Phys. Rev. Lett. 1995. V. 74. P. 2863; D. V. Gal'tsov, O. V. Kechkin. Phys. Lett. B. 1995. V. 361. P. 52; Phys. Rev. D. 1996. V. 54. P. 1656; D. V. Gal'tsov, S. A. Sharakin. Phys. Lett. B. 1997. V. 399. P. 250.

[16] A. Das, J. Maharana, A. Melikyan. Monodromy, duality and integrability of two dimensional string effective action. hep-th/0210012.

[17] Г. А. Алексеев. Интегрируемость обобшенных (матричных) уравнений Эрнста в теории струн. ТМФ. 2005 (в печати); hep-th/0410246.

[18] H. Stephani, D. Kramer, M. MacCallum, C. Hoenselaers, E. Herlt. Exact Solutions of Einstein's Field Equations. 2nd edition. Cambridge: Cambridge Univ. Press, 2003.

[19] V. Belinski, E. Verdaguer. Gravitational Solitons. Cambridge: Cambridge Univ. Press, 2001.

[20] Г. А. Алексеев. Тр. МИАН СССР. 1987. Т. 176. № 4. С. 211.

[21] H. Р. Сибгатуллин. Колебания и волны в сильных гравитационных и электромагнитных полях. М.: Наука, 1984.

[22] G. Neugebauer. Phys. Lett. A. 1981. V. 86. № 2. P. 91.

[23] Г. А. Алексеев. ДАН СССР. 1985. Т. 283. № 3. С. 577.

[24] G. A. Alekseev. Monodromy transform approach to solution of some field equations in general relativity and string theory. In: Proc. of Workshop on "Nonlinearity, Integrability and all that: Twenty Years after NEEDS'79". Eds. M. Boiti et al. Singapore: World Scientific, 2000. P. 12; gr-qc/9911045.

[25] G. A. Alekseev. Explicit form of the extended family of electrovacuum solutions with arbitrary number of parameters. In: Abstracts of Contributed Papers of 13th Intern. Conf. on General Relativity and gravitation. Eds. P. W. Lamberti, O. E. Ortiz. Cordoba, Argentina: Huerta Grande, 1992. P. 3.

[26] G. A. Alekseev, A. A. Garcia. Phys. Rev. D. 1996. V. 53. P. 1853.

[27] G. A. Alekseev. Integrability of the boundary value problems for the Ernst equations. In: Proc. of Workshop "Nonlinear Evolution Equations and Dynamical Systems" (Dubna, 1992). Eds. V. Makhankov et al. Singapore: World Scientific, 1993. P. 5.

[28] F. J. Ernst. Phys. Rev. 1968. V. 167. № 2. P. 1175; V. 168. № 2. P. 1415.

[29] I. Hauser, F. J. Ernst. J. Math. Phys. 1980. V. 21. P. 1126.

[30] I. Hauser, F. J. Ernst. Gen. Rel. Grav. 2001. V. 33. P. 195.

[31] Н. И. Мусхелишвили. Сингулярные интегральные уравнения. М.: Наука, 1968.

[32] Ф. Д. Гахов. Краевые задачи. М.: Наука, 1977. 